\title{
Regular dependence of invariant curves and Aubry-Mather sets of twist maps of an annulus
}

\author{
RAPHAËL DOUADY* \\ Ecole Polytechnique, Centre de Mathématiques, 91128 Palaiseau Cedex, France
}

(Received 29 July 1986 and revised 30 June 1987)

Abstract. We prove that smooth enough invariant curves of monotone twist maps of an annulus with fixed diophantine rotation number depend on the map in a differentiable way. Partial results hold for Aubry-Mather sets.

Then we how that invariant curves of the same map with dif srent rotation numbers $\omega$ and $\omega^{\prime}$ cannot approach each other at a distance less than cst. $\left|\omega-\omega^{\prime}\right|$. By K.A.M. theory, this implies that, under suitable assumptions, the union of invariant curves has positive measure.

Analogous results are due to Zehnder and Herman (for the first part), and to Lazutkin and Pöschel (for the second one), in the case of Hamiltonian systems and area preserving maps.

1. Introduction

Let $f: \mathbb{T}^{1} \times[-\delta, \delta] \rightarrow \mathbb{T}^{1} \times[-\delta, \delta]$

$$
(\theta, r) \mapsto(\Theta, R),
$$

(where $\mathbb{J}^{1}=\mathbb{R} / \mathbb{Z}$ ) be a diffeomorphism which is isotopic to the identity map and satisfies the monotone twist condition:

$$
\partial \Theta / \partial r>0 \text {. }
$$

Let $\omega_{+}$(resp. $\omega_{-}$) be the rotation number of $\left.f\right|_{T^{1} \times\{\delta\}}$ (resp. $\left.f\right|_{\mathbb{T}^{1} \times\{-\delta\}}$ ).

Mather [10] and Aubry-Le Daeron [1] (see also Chenciner [3] and Hedlund [4]) proved that, if $f$ preserves area, then for any $\omega \in\left[\omega_{-}, \omega_{+}\right]$, there exists an orbit of $f$ :

$$
\left(\theta_{n}, r_{n}\right)=f^{n}\left(\theta_{0}, r_{0}\right), \quad n \in \mathbb{Z}
$$

and a (non-necessarily continuous) map $h_{\omega}: \mathbb{T}^{1} \rightarrow \mathbb{T}^{1}$ preserving cyclic order and such that:

$$
h_{\omega}(n \omega)=\theta_{n}
$$

holds for every integer $n$. When $\omega$ is irrational, the closure $\Gamma_{\omega}$ of this orbit is the graph of a Lipschitz map:

where

$$
\psi_{\omega}: K_{\omega} \rightarrow[-\delta, \delta]
$$

$$
K_{\omega}=\overline{\left\{\theta_{n}, n \in \mathbb{Z}\right\}}
$$

* Partially supported by U.A. du CNRS no. 169: MSRI, Berkeley California, USA and IMPA/CNPq, Rio de Janeiro, Brasil. 
The set $\Gamma_{\omega}$ is an invariant curve if $K_{\omega}=\mathbb{T}^{1}$, and a totally discontinuous set otherwise. In the first case, $h_{\omega}$ is a homeomorphism of $\pi^{1}$ satisfying

$$
f\left(h_{\omega}(\theta), \psi_{\omega} \circ h_{\omega}(\theta)=\left(h_{\omega}(\theta+\omega), \psi_{\omega} \circ h_{\omega}(\theta+\omega)\right)\right.
$$

and $\psi_{\omega}$ is uniquely determined; so is $h_{\omega}$ provided that one requires the normalization

$$
h_{\omega}(0)=0 \text {. }
$$

The problem of knowing for which values of $\omega$, the set $\Gamma_{\omega}$ is a curve and, in this case, of determining the smoothness of $\psi_{\omega}$ and $h_{\omega}$ is very difficult (see Moser [11], Rüssmann [13], Herman [5,7]). The only cases where $\Gamma_{\omega}$ is known to be a curve occur when $\omega$ satisfies a diophantine condition (see $\S 2$ ) and $f$ is differentiable enough.

Remark. By Herman theorem ([5]) on differentiable conjugation of diffeomorphisms of the circle to rotations (generalized by Yoccoz [14]) if $\Gamma_{\omega}$ is a smooth curve, and if $\omega$ is diophantine, then $h_{\omega}$ is smooth.

In this paper, we study, for fixed diophantine $\omega$, the regular dependence of the set $\Gamma_{\omega}$ on the mapping $f$. Our results actually do not need that $f$ preserves area. Then we give a lower bound to the distance separating two different invariant curves $\Gamma_{\omega}$ and $\Gamma_{\omega}$ differentiable enough, of the same mapping $f$, which is proportional to $\left|\omega-\omega^{\prime}\right|$. We also study the Whitney-differentiable dependance of $\Gamma_{\omega}$, on $\omega$.

In $\S 3$, we prove the elementary properties of the Farey series we need in the following two sections.

$\S \S 4$ and 5 study the case where $f$ is considered in the $C^{0}$-topology (with bounded $C^{1}$-norm). If, for the smooth mapping $f$, the set $\Gamma_{\omega}\left(f_{0}\right)$ is a smooth curve (actually finite differentiability is enough, the order depending in the diophantine exponent $2+\beta$ of $\omega)$, then, for $f C^{1}$-close to $f_{0}$, the Hausdorff distance between the sets $\Gamma_{\omega}\left(f_{0}\right)$ and $\Gamma_{\omega}(f)$ is not more than

$$
\text { cst. }\left\|f-f_{0}\right\|_{C^{0}}^{\tau}, \text { with } \tau=\frac{2+\beta}{3+2 \beta} \in\left(\frac{1}{2}, \frac{2}{3}\right] \text {. }
$$

This result is stated at the end of $\S 6$, which is devoted to normal forms.

In $\S 7$, we prove that the map $f \mapsto \Gamma_{\omega}(f)$ is differentiable at $f_{0}$ (in a sense to be defined), when $f$ is considered in the $C^{l}$-topology, for $l>3+2 \beta$.

The idea, to prove this last statement, is to look for an approximative location of $\Gamma_{\omega}(f)$, through normal forms, which would allow us to square the size of the perturbation. Then, we apply the previous theorem and use the crucial fact that $\tau>\frac{1}{2}$.

We show, in $\S 8$, how it is possible to lower the minimal value of $l$, using smoothing operators in our changes of variables. This idea is, actually, the basic tool of Hamilton's implicit function theorem (see [2]).

By taking larger values of $l$ and making further normal forms, one could prove that, if $f_{0}$ and $\Gamma_{\omega}\left(f_{0}\right)$ are smooth, then the map $f \mapsto \Gamma_{\omega}(f)$ admits Taylor expansions of arbitrary order at $f_{0}$.

Analogous results come out of Herman's and Zehnder's proofs (see $[2,6,15]$ ) of the existence of invariant tori for Hamiltonian systems, and of translated curves for monotone twist maps, which use the implicit function theorem in Frechet spaces. 
In the last section, we show that if $\Gamma_{\omega}$ is a smooth invariant curve of a smooth twist map $f$, then any Aubry-Mather set $K_{\omega^{\prime}}$ with rotation number $\omega^{\prime} \neq \omega$ of $f$ cannot approach $\Gamma_{\omega}$ at a distance less than cst. $\left|\omega-\omega^{\prime}\right|$ (again, finite differentiability is enough).

This implies, by the Kolmogorov-Arnol'd-Moser theorem, that the union of the invariant curves has positive Lebesgue measure.

We also prove that, when $\omega$ varies in a set of diophantine numbers with fixed exponent and constant, the map $\omega \mapsto \Gamma_{\omega}$ is Whitney-differentiable (here, $\Gamma_{\omega}$ has to be considered in the $C^{0}$-topology).

This result was known to Lazutkin [9] in the case of caustics of a convex billiards and to Pöschel [12] for Hamiltonian systems. The interest of our method is that it is completely geometric and does not use functional analysis.

This is probably why we also get results for Aubry-Mather sets, but we only control the $C^{0}$-norm of invariant curves.

\section{Acknowledgements}

The author would like to thank Jean-Christophe Yoccoz who told him about the existence of Farey series.

Besides, it is a pleasure to the author to express his gratitude to M.S.R.I. and I.M.P.A. who offered him the best working conditions.

\section{Notations}

Let $\mathbb{T}^{1}=\mathbb{R} / \mathbb{Z}, A=\mathbb{T}^{1} \times \mathbb{R}$ and, for $\delta \geq 0, \mathbb{A}_{\delta}=\mathbb{T}^{1} \times[-\delta, \delta]$. When $\theta \in \mathbb{T}^{1}$, we set

$$
\|\theta\|=\inf _{p \in \mathbf{Z}}|\tilde{\theta}-p|
$$

where $\tilde{\theta}$ is any lift of $\theta$ in $\mathbb{R}$. If $(\theta, r) \in \mathbb{A}$, then:

$$
\|(\theta, r)\|=\sqrt{\|\theta\|^{2}+r^{2}}
$$

When $x \in \mathbb{R},[x]$ denotes the integer part of $x:[x] \leq x<[x]+1$.

Let $M=\mathbb{T}^{1}$ or $\mathbb{A}_{\delta}$, and $k \in \mathbb{N}$. The space $C^{k}(M)=C^{k}(M, \mathbb{R})$ is provided with the norm:

$$
\|\varphi\|_{C^{k}}=\sup _{0 \leq j \leq k} \max _{M}\left|D^{j} \varphi\right| .
$$

If $k \in \mathbb{R}_{+} \backslash \mathbb{N}$, and $k=k_{0}+\alpha, k_{0} \in \mathbb{N}, 0<\alpha<1$, then $C^{k}(M)$ denotes the subspace of $C^{k_{o}}(M)$ containing the function $\varphi$ whose $k_{0}$ th derivative $D^{k_{0}} \varphi$ satisfies a Hölder condition of order $\alpha$. Its norm is:

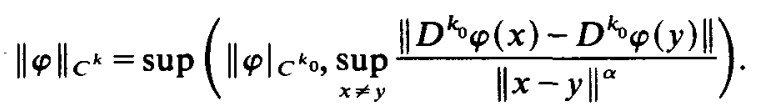

For $k \in[1,+\infty], \operatorname{Diff}_{+}^{k}\left(\mathbb{T}^{1}\right)$ denotes the group of order-preserving $C^{k}$ diffeomorphisms of $\mathbf{T}^{1}$.

When $\varphi \in C^{k}\left(\mathbb{J}^{1}\right), \varphi^{\prime}$ (resp. $\varphi^{\prime \prime}, \ldots$ ) will denote its derivative (resp. second derivative, ...).

We shall not make any distinction between functions defined on $\mathbb{T}^{1}$ and 1-periodic functions defined on $\mathbb{R}$. 
When $f: M \rightarrow M$, (resp. $f$ is $1-1$ ), we write $f^{n}$ for the $n$th iterate of $f, n \in \mathbb{N}$ (resp. $n \in \mathbb{Z})$.

If $f$ and $g$ are $C^{k}$-imbeddings of $A_{\delta}$ into $A$, and if $g=f+(u(\bmod 1), v)$, with $u$, $v \in C^{k}\left(A_{\delta}\right)$, then the $C^{k}$-distance between $f$ and $g$ is defined as

$$
\|g-f\|_{C^{k}}=\sup \left(\inf _{p \in \mathbf{Z}}\|u-p\|_{C^{k}},\|v\|_{C^{k}}\right) \text {. }
$$

Let $h$ be an order preserving homeomorphism of a closed subset $K \subset \mathbb{R}$ such that:

- $K$ is invariant under $x \mapsto x+1$

$-h(x+1)=h(x)+1 \quad$ for every $x \in K$.

The number:

$$
\rho(h)=\lim _{n \rightarrow+\infty} \frac{1}{n} h^{n}(x)
$$

does not depend on the initial point $x$ chosen in $K$, and is called the rotation number of $h$.

When $h$ is a homeomorphism preserving cyclic order of a compact set $K \subset \mathbb{J}^{1}$, its rotation number is defined in $\mathbb{T}^{1}$ as the class $(\bmod 1)$ of the rotation number of any order preserving lift $\tilde{h}$ of $h$ to the preimage $\tilde{K}$ of $K$ in $\mathbb{R}$.

An irrational number $\omega$ satisfies a diophantine condition with exponent $2+\beta, \beta \geq 0$ and constant $\gamma>0$ if:

$$
\left|\omega-\frac{p}{q}\right| \geq \frac{\gamma}{q^{2+\beta}}
$$

holds for every $(p, q) \in \mathbb{Z} \times \mathbb{Z}_{+}$.

\section{Farey series}

Let $c$ be a positive integer.

Definition. The Farey series of order $c$ is the set:

$$
F_{c}=\left\{\frac{p}{q} \in \mathbb{Q} /(p, q) \in \mathbb{Z}^{2} \text { and } 0<q \leq c\right\} .
$$

Obviously, $F_{c}$ is closed, discrete and contains $F_{c-1}$. When we write $p / q \in F_{c}$, we shall assume that $p$ and $q$ are relatively prime integers and that $0<q \leq c$.

Proposition 0. Let $p / q$ and $p^{\prime} / q^{\prime}$ be two consecutive elements of $F_{c}$, then the following hold:

$$
\begin{aligned}
& q+q^{\prime} \geq c+1, \\
& \frac{p^{\prime}}{q^{\prime}}-\frac{p}{q}=\frac{1}{q q^{\prime}} .
\end{aligned}
$$

Proof. Inequality (i) follows easily from the framing:

$$
\frac{p}{q}<\frac{p+p^{\prime}}{q+q^{\prime}}<\frac{p^{\prime}}{q^{\prime}}
$$

For (ii), we have to show that the sublattice $\Gamma$ of $\mathbb{Z}^{2}$ spanned by $(p, q)$ and $\left(p^{\prime}, q^{\prime}\right)$ has volume 1 . By assumption, there is no integral point, but the vertices, in the triangle $(0,0),(p, q)$ and $\left(p^{\prime}, q^{\prime}\right)$ : such a point would give rise to an element of $F_{c}$ 
between $p / q$ and $p^{\prime} / q^{\prime}$. From the symmetry $(x, y) \mapsto\left(p+p^{\prime}-x, q+q^{\prime}-y\right)$, we deduce that there is no integral point, but the vertices, in the parallelogram $(0,0),(p, q)$, $\left(p^{\prime}, q^{\prime}\right)$ and $\left(p+p^{\prime}, q+q^{\prime}\right)$, hence $\Gamma$ as volume 1 .

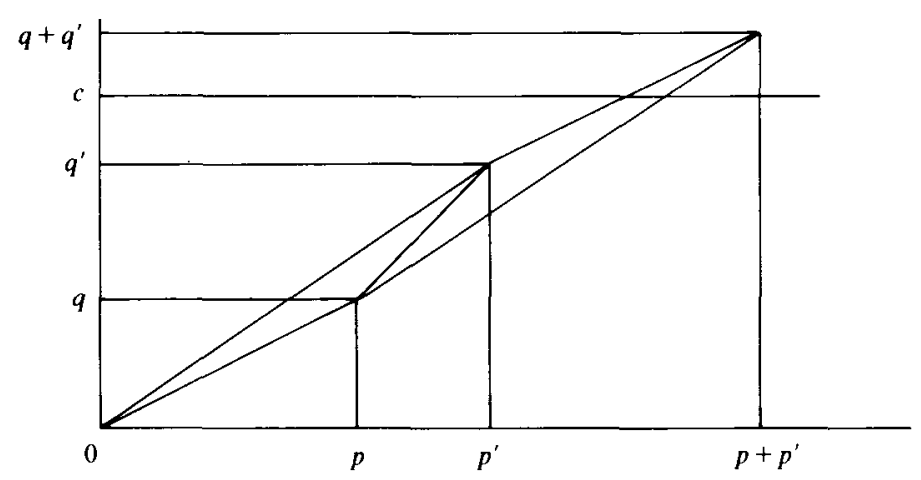

\section{Hölder dependence of invariant curves}

\section{1 .}

Let $\delta>0$ and $f: A_{\delta} \rightarrow A$ be a $C^{1}$-embedding which takes the following form, where $f(\theta, r)=(\Theta, R)$ :

$$
\left\{\begin{array}{l}
\Theta=\theta+\omega+r a(\theta, r) \quad(\bmod 1) \\
R=\lambda r+v(\theta, r)
\end{array}\right.
$$

We suppose that the scalar $\lambda$ satisfies $\lambda \geq 1$, while $a$ and $v$ belong to $C^{0}\left(A_{\delta}\right)$, the function $a$ being positive everywhere. The real number $\omega$ is irrational and satisfies a diophantine condition with exponent $2+\beta$ and constant $\gamma$ (see $\S 2$ ).

Notice that, if $v(\theta, 0) \equiv 0$, then $\mathbb{A}_{0}=\mathbb{T}^{1} \times\{0\}$ is an invariant circle, and $f_{\mid A_{0}}$ is simply a rotation by $\omega$.

We shall suppose, in this section, that there exists a continuous function $\psi: \nabla^{1} \rightarrow$ $[-\delta, \delta]$, whose graph $\Gamma$ is invariant $\dagger$ under $f$ and such that $f_{\mid \Gamma}$ is $C^{1}$-conjugated to the rotation $\theta \rightarrow \theta+\omega$ (actually, a more precise assumption will be made on a lift $\tilde{f}$ of $f$ to $\left.\tilde{\mathbb{A}}_{\delta}=\mathbb{R} \times[-\delta, \delta]\right)$.

The problem is to estimate $\|\psi\|_{C^{0}}$ in terms of $\|v\|_{C^{0}}$ and other quantities involving $a$ and $\omega$.

Let us resume the assumptions. We denote by $\tilde{f}: \mathbb{R} \times[-\delta, \delta] \rightarrow \mathbb{R}^{2},(\tilde{\theta}, r) \rightarrow(\tilde{\Theta}, R)$, the unique lift of $f$ satisfying $\tilde{\Theta}(\tilde{\theta}, 0)=\tilde{\theta}+\omega$ (remember that $\omega \in \mathbb{R})$.

There exist functions $\psi \in C^{0}\left(\mathbb{T}^{1}\right)$ and $\eta \in C^{1}\left(\mathbb{T}^{1}\right)$ such that:

(i) $\tilde{h}=\operatorname{Id}_{\mathbb{R}}+\eta \in \operatorname{Diff}_{+}^{1}(\mathbb{R})$, that is $\eta^{\prime}>-1$.

(ii) $\|\psi\|_{C^{0}} \leq \delta$. The graph $\Gamma$ of $\psi$ is hence included in $\mathbb{A}_{\delta}$.

(iii) $\tilde{f}(\tilde{h}(\theta), \psi \circ \tilde{h}(\theta))=(\tilde{h}(\tilde{\theta}+\omega), \psi \circ \tilde{h}(\tilde{\theta}+\omega))$ holds for every $\tilde{\theta} \in \mathbb{R}$. We denote by $h$ the diffeomorphism of $\mathbb{T}^{1}$ induced by $\tilde{h}$. Notice that condition (iii) implies

† From Birkhoff's result (see Herman [7]), if $f$ preserves area and if $\partial \Theta / \partial r>0$, then any curve which is invariant under $f$ and homotopic to $\mathbf{A}_{\mathbf{0}}$, is the graph of a Lipschitz function. 
that the rotation number of $\tilde{f}$ restricted to the lift $\tilde{\Gamma}$ of $\Gamma$ in $\tilde{A}_{\delta}=\mathbb{R} \times[-\delta, \delta]$ is $\omega$ (expecting any control on an invariant curve with rotation number $\omega+p, p \in \mathbb{Z} \backslash\{0\}$, would be absurd).

4.2.

To simplify notations, let us set:

$$
\begin{aligned}
a_{-} & =\min a>0, \\
A & =\max a=\|a\|_{C^{0}}, \\
V & =\|v\|_{C^{0}}, \\
H & =\left\|\eta^{\prime}\right\|_{C^{0}} \leq\|\eta\|_{C^{1}}, \\
\rho & =\|\psi\|_{C^{0}} .
\end{aligned}
$$

Proposition 1. One has:

$$
\rho \leq H / 2 a_{-}
$$

and, if $\mathrm{Va} a_{-} \leq 2 H$, then:

$$
\rho \leq \frac{(2 H)^{1-\tau} V^{\tau}}{\gamma^{1 /(3+2 \beta)} a_{-}^{1-\tau}}
$$

with $\tau=(2+\beta) /(3+2 \beta)$.

4.3. Remark.

We give here an estimate of $\rho$ which we mean to be independent of $\lambda$. If one knows that $\lambda>1$, then it is not hard to get the majoration:

$$
\rho \leq \frac{V}{\lambda-1}
$$

since a point whose $r$ coordinate would exceed $V /(\lambda-1)$ in absolute value would have to leave $A_{\delta}$ in a finite time.

\subsection{Proof}

We can assume, without loss of generality, that $h(0)=0$ and that $\psi(0)=\rho>0$. From now on, we shall work in the universal covering $\tilde{A}_{\delta}=\mathbb{R} \times[-\delta, \delta]$ of $\mathbb{A}_{\delta}$, so that inequalities in the $\theta$ coordinate are meaningful.

From (iii), one gets:

$$
|\psi \circ h(\theta+\omega)-\lambda \psi \circ h(\theta)| \leq V
$$

for every $\theta \in \mathbb{R}$. Hence, by an easy induction, and since $\lambda \geq 1$, the inequality:

$$
\psi \circ h(k \omega) \geq \rho-k V
$$

holds for any integer $k \leq \rho / V$. On the other hand,

$$
\tilde{h}(\theta+\omega)-\tilde{h}(\theta)-\omega=\psi \circ \tilde{h}(\theta) a(\tilde{h}(\theta), \psi \circ \tilde{h}(\theta))
$$

hence

$$
\tilde{h}((k+1) \omega)-\tilde{h}(k \omega)-\omega \geq(\rho-k V) a_{-} .
$$

A summation over $k$ gives

$$
\eta(k \omega)=\tilde{h}(k \omega)-k \omega \geq k \rho a_{-}-\frac{k(k-1)}{2} V a_{-} .
$$


From this inequality, and since $\eta(0)=0$, we deduce that

$$
\begin{aligned}
\rho a_{-} & \leq(k-1) \frac{V A}{2}+\frac{1}{k} \eta(k \omega) \\
& \leq(k-1) \frac{V A}{2}+H \frac{\|k \omega\|}{k}
\end{aligned}
$$

holds for every $k \leq \rho / V$.

Replacing $k$ by 1 in this inequality leads to the first inequality of Proposition 1 (remember that $\|\omega\| \leqq \frac{1}{2}$ ).

Let $z=V a_{-} / 2 H$ and suppose now that $z \leq 1$. Let

$$
c=\left[\gamma^{-1 /(3+2 \beta)} z^{-(1+\beta) /(3+2 \beta)}\right] \geq 1,
$$

(where $[x]$ denotes the integer part of $x$ ). If $\rho \leq c V$, then the second inequality holds. Otherwise we consider two consecutive elements $p / q$ and $p^{\prime} / q^{\prime}$, of the Farey series $F_{c}$ of order $c$ such that:

$$
p / q<\omega<p^{\prime} / q^{\prime}
$$

One has, from Proposition 0:

$$
\frac{1}{q q^{\prime}}=\frac{p^{\prime}}{q^{\prime}}-\frac{p}{q}=\left(\frac{p^{\prime}}{q^{\prime}}-\omega\right)+\left(\omega-\frac{p}{q}\right) \geq \gamma\left(\frac{1}{q^{2+\beta}}+\frac{1}{q^{2+\beta}}\right),
$$

hence:

$$
\left(q q^{\prime}\right)^{1+\beta} \geq \gamma\left(q^{2+\beta}+q^{2+\beta}\right) .
$$

From the convexity of $x \mapsto x^{2+\beta}$, and from $q+q^{\prime} \geq c+1$, we obtain:

$$
q q^{\prime} \geq \frac{1}{2} \gamma^{1 /(1+\beta)}(c+1)^{(2+\beta) /(1+\beta)} \text {. }
$$

Consequently,

$$
\begin{aligned}
\inf \left(\frac{p^{\prime}}{q^{\prime}}-\omega, \omega-\frac{p}{q}\right) \leq \frac{1}{2 q q^{\prime}} & \leq \gamma^{-1 /(1+\beta)}(c+1)^{-(2+\beta) /(1+\beta)} \\
& \leq \gamma^{-1 /(3+2 \beta)} z^{(2+\beta) /(3+2 \beta)} .
\end{aligned}
$$

On the other hand,

$$
\sup \left(z q, z q^{\prime}\right) \leq z c \leq \gamma^{-1 /(3+2 \beta)} z^{(2+\beta) /(3+2 \beta)} .
$$

The second inequality of Proposition 1 follows from these two estimates and from the choice of $k=q$ or $q^{\prime}$ in (4.4.1).

\section{Hölder dependence of Aubry-Mather sets}

\section{1.}

Here, the setting is the same as in $\$ 4.1$, but, instead of an invariant curve, we only assume that $f$ possesses an invariant closed set to which the restriction of $f$ has rotation number $\omega$. Precisely, there is a closed subset $K$ of $\mathbb{T}^{1}$ and a continuous function $\psi: K \rightarrow[-\delta, \delta]$ such that, denoting by $\tilde{K}$ the preimage:of $K$ in $\mathbb{R}$ and by $\tilde{f}$ the lift of $f$ defined in 4.1, and defining:

$$
\begin{aligned}
g: \tilde{K} & \rightarrow \mathbb{R} \\
\theta & \mapsto \theta+\omega+\psi(\theta) a(\theta, \psi(\theta))
\end{aligned}
$$


then:

(i) $g(\tilde{K})=\tilde{K}$ and $g$ is an increasing homeomorphism of $\tilde{K}$ whose rotation number is $\omega$ (obviously, $g(\theta+1)=g(\theta)+1)$.

(ii) $\tilde{f}(\theta, \psi(\theta))=(\tilde{g}(\theta), \quad \psi \circ g(\theta))$ holds for every $\theta \in \tilde{K}$.

We set: $\rho=\max _{K}|\psi|$; quantities $a_{-}, A$ and $V$ were defined in 4.2.

5.2 .

Proposition 2. One always has:

$$
\rho \leq 1 / a_{-}
$$

and, if $V a_{-} \leq 2$, then:

$$
\rho \leq \frac{3}{a_{-}^{1-\tau}}\left(\frac{V}{2}\right)^{\tau} \gamma^{-1 /(3+2 \beta)},
$$

where $\tau=(2+\beta) /(3+2 \beta)$.

Remarks. 1. The second inequality of Proposition 2 is sharper than the inequality of Proposition 1 if

$$
H \geq\left(\frac{3}{2}\right)^{(3+2 \beta) /(1+\beta)} .
$$

2. Remark 4.3 is still available here.

Proof. It is very similar to that of Proposition 1 . We may assume that $0 \in \tilde{K}$ and that $\rho=\psi(0)$.

For any $\theta \in K$, one has:

$$
\psi \circ g(\theta) \geq \lambda \psi(\theta)-V
$$

hence, if:

$$
\begin{aligned}
& \theta_{k}=g^{k}(0) \\
& \rho_{k}=\psi\left(\theta_{k}\right), \quad k=0,1,2, \ldots,
\end{aligned}
$$

then as $\lambda \geq 1$, the inequality:

$$
\rho_{k} \geq \rho-k V
$$

holds for every integer $k \leq \rho / V$. An easy induction gives:

$$
\begin{aligned}
\theta_{k} & =k \omega+\rho_{0} a\left(\theta_{0}, \rho_{0}\right)+\cdots+\rho_{k-1} a\left(\theta_{k-1}, \rho_{k-1}\right) \\
& \geq k \omega+k \rho a_{-} \frac{k(k-1)}{2} V a_{-} .
\end{aligned}
$$

Remember that the rotation number of $g$ is $\omega$, that is, for every $(p, q) \in \mathbb{Z}^{2}$, and $\theta \in K, q \omega-p$ and $g^{q}(\theta)-\theta-p$ have the same sign (they only vanish if $p=q=0$ ). Let $(p, q) \in \mathbb{Z} \times \mathbb{Z}_{+}$be such that $p / q>\omega$, then $\theta_{q}<p$ and:

$$
\rho a_{-}<\frac{p}{q}-\omega+(q-1) \frac{V a_{-}}{2} .
$$

Choosing $q=1$ and $p=[\omega]+1$ leads to the first inequality.

Let us assume now that $z=V a_{-} / 2 \leq 1$, and set

$$
c=\left[\gamma^{-1 /(3+2 \beta)} z^{-(1+\beta) /(3+2 \beta)}\right] \text {. }
$$


If $\rho \leq c V$, then the second inequality holds. Otherwise, let us consider two consecutive elements $p / q$ and $p^{\prime} / q^{\prime}$ of the Farey series $F_{c}$ of order $c$ such that

One has:

$$
p^{\prime} / q^{\prime}<\omega<p / q .
$$

$$
\frac{1}{q q^{\prime}}=\frac{p}{q}-\frac{p^{\prime}}{q^{\prime}}=\left(\frac{p}{q}-\omega\right)+\left(\omega-\frac{p^{\prime}}{q^{\prime}}\right) \geq \gamma\left(\frac{1}{q^{2+\beta}}+\frac{1}{q^{\prime 2+\beta}}\right)
$$

hence,

$$
\left(q q^{\prime}\right)^{1+\beta} \geq \gamma\left(q^{2+\beta}+q^{2+\beta}\right) .
$$

By convexity of $x \mapsto x^{2+\beta}$ and since $q+q^{\prime} \geq c+1$, one has:

$$
q q^{\prime} \geq \frac{1}{2} \gamma^{1 /(1+\beta)}(c+1)^{(2+\beta) /(1+\beta)}
$$

hence

$$
\frac{p}{q}-\omega \leq \frac{1}{q q^{\prime}} \leq 2 \gamma^{-1 /(1+\beta)}(c+1)^{-(2+\beta) /(1+\beta)} \leq 2 \gamma^{-1 /(3+2 \beta)} z^{(2+\beta) /(3+2 \beta)} .
$$

On the other hand,

$$
q z \leq c z \leq \gamma^{-1 /(3+2 \beta)} z^{(2+\beta) /(3+2 \beta)} .
$$

Together, these two estimates give the result.

\section{Reduction of monotone twist diffeomorphisms}

6.1.

Let $f: \mathbb{A}_{\delta} \rightarrow \mathbb{A}$

$$
(\theta, r) \mapsto(\Theta, R)
$$

be an imbedding of class $C^{k}, k \geq 1$, satisfying the monotone twist condition:

$$
\partial \Theta / \partial r>0 \text {. }
$$

We assume that there exists mappings $\psi \in C^{k}\left(\mathbb{T}^{1}\right)$ and $h \in \operatorname{Diff}_{+}^{k}\left(\mathbb{T}^{1}\right)$, and a real number $\omega$ satisfying a diophantine condition with exponent $2+\beta$ and constant $\gamma$, such that $\|\psi\|_{C^{0}<\delta \text { and that: }}$

$$
f(h(\theta)), \psi \circ h(\theta))=(h(\theta+\omega), \psi \circ h(\theta+\omega))
$$

holds for every $\theta \in \mathbb{T}^{1}$. This means that the graph $\Gamma$ of $\psi$ is invariant under $f$ and that the restriction $f_{\mid \Gamma}$ is conjugated, by the action of $h$ on the first coordinate, to the rigid rotation $\theta \mapsto \theta+\omega$.

If we replace $f$ by $H \circ f \circ H^{-1}$, where the $C^{k}$-diffeomorphism $H$ is defined by:

$$
H(\theta, r)=\left(h^{-1}(\theta), r-\psi(\theta)\right),
$$

we come down to a situation where $\psi=0$ and $h=\mathrm{Id}_{\mathrm{T}^{1}}$. Moreover, the monotone twist condition is still satisfied, since $H$ preserves the "verticals" $\{\theta=\operatorname{cst}\}$, and $H \circ f \circ H^{-1}$ is defined on the set $H\left(\mathbb{A}_{\delta}\right)$ which contains the annulus $\mathbb{A}_{\delta^{\prime}}$, with $\delta^{\prime}=\delta-\|\psi\|_{C^{0}}$.

Henceforth, we suppose that $\psi=0$ and $h=\operatorname{Id}_{\mathbb{T}^{1}}$. The map $f$ then takes the following form:

$$
\left\{\begin{array}{l}
\Theta=\theta+\omega+r a(\theta, r) \quad(\bmod 1) \\
R=r \lambda(\theta, r)
\end{array}\right.
$$

where $a$ and $\lambda$ are positive functions of class $C^{k-1}$ on $A_{\delta}$. 
6.2.

The next reduction needs a lemma on difference equations.

LEMMA. Let $\kappa \in \mathbb{P}_{\mp}$ such that $\kappa>1+\beta$ and, neither $\kappa$ nor $\kappa-1-\beta$ are integers, and let $\nu>0$ and $\varphi \in C^{\kappa}\left(\mathbb{T}^{1}\right)$ satisfy:

$$
\int_{0}^{1} \varphi(\theta) d \theta=0
$$

There exists $\eta \in C^{\kappa-1-\beta}\left(\mathbb{T}^{1}\right)$ (unique up to a constant if $\nu=1$, and unique otherwise) such that

$$
\eta(\theta+\omega)-\nu \eta(\theta)=\varphi(\theta),
$$

holds for every $\theta \in \mathbb{T}^{1}$. Moreover, ther exists a constant $C$ depending only on $\kappa$ and $\beta$ such that, if one assumes

$$
\int_{0}^{1} \eta(\theta) d \theta=0
$$

when $\nu=1$, then:

$$
\|\eta\|_{C^{\kappa-1-\beta}} \leq \frac{C}{\gamma}\|\varphi\|_{C^{\kappa}}
$$

(We recall that $\omega$ is supposed to be diophantine with exponent $2+\beta$ and constant r.)

A proof of this lemma is given in Herman [5, p. 229].

Assume now that $k>3+\beta$ and $k \notin \mathbb{N} \cup(\beta+\mathbb{N})$. The lemma furnishes a positive function $\mu \in C^{k-2-\beta}\left(\mathbb{T}^{1}\right)$ satisfying:

$$
\frac{\mu(\theta)}{\mu(\theta+\omega)}=\frac{1}{\bar{\lambda}} \lambda(\theta, 0)
$$

for every $\theta \in \mathbb{T}^{1}$, where the scalar $\bar{\lambda}$ is defined by

$$
\log \bar{\lambda}=\int_{0}^{1} \log \lambda(\theta, 0) d \theta .
$$

Let $H_{1}$ be the $C^{k-1-\beta}$-diffeomorphism of $A$ defined by:

$$
H_{1}(\theta, r)=\left(\theta, \int_{\tilde{\theta}}^{\tilde{\theta}+r} \mu(t) d t\right) \stackrel{\text { def }}{=}(\bar{\theta}, \bar{r}),
$$

where $\tilde{\theta}$ is any left of $\theta$ in $\mathbb{R}$. The set $H_{1}\left(\mathbb{A}_{\delta}\right)$ contains the annulus $A_{\bar{\delta}}$, with $\bar{\delta}=\delta \min \mu$.

One now easily checks that $\bar{f}=H_{1} \circ f \circ H_{1}^{-1}$ satisfies the monotone twist condition (since $\bar{\theta}=\theta)$ and, if $\bar{f}(\bar{\theta}, \bar{r})=(\bar{\Theta}, \bar{R})$, then:

$$
\left\{\begin{array}{l}
\bar{\Theta}=\bar{\theta}+\omega+\bar{r} \bar{a}(\bar{\theta}, \bar{r}) \quad(\bmod 1) \\
\bar{R}=\bar{\lambda} \bar{r}+\bar{r}^{2} b(\bar{\theta}, \bar{r})
\end{array}\right.
$$

where $\bar{a}$ is $C^{k-2-\beta}$ and positive everywhere, $b$ is $C^{k-3-\beta}$ and $\bar{\lambda}$ was defined above. Furthermore, one has:

$$
\bar{a}_{-}=\min \bar{a} \geq \min a / \max \mu .
$$


6.3.

We now consider a $C^{\prime}$-perturbation $f^{\prime}$ of $f$, with $1 \leq l \leq k$, and set $\bar{f}^{\prime}=H_{1} \circ f^{\prime} \circ H_{1}^{-1}$. We now assume that $l \leq k-2-\beta$. There exists a constant $C_{1}$ depending only on $f$, $\omega$ and $l$ such that

$$
\left\|\bar{f}^{\prime}-\bar{f}\right\|_{C^{\prime}} \leq C_{1}\left\|f^{\prime}-f\right\|_{C^{\prime}}
$$

since $H_{1}$ is $C^{l+1}$.

Let us set $\bar{f}^{\prime}(\bar{\theta}, \bar{r})=\left(\bar{\Theta}^{\prime}, \bar{R}^{\prime}\right)$, and

$$
\left\{\begin{array}{l}
\left.\bar{\Theta}^{\prime}=\bar{\Theta}+u(\bar{\theta}, \bar{r}) \quad \bmod 1\right) \\
\bar{R}^{\prime}=\bar{R}+v(\bar{\theta}, \bar{r})
\end{array}\right.
$$

where $u$ and $v$ belong to $C^{\prime}\left(A_{\delta}\right)$. If the norm $\|u\|_{C^{1}}$ is sufficiently small, then $f^{\prime}$ is still a monotone twist diffeomorphism, and the map:

$$
H_{2}:(\bar{\theta}, \bar{r}) \mapsto\left(\bar{\theta}, \bar{r}+\frac{u(\bar{\theta}, \bar{r})}{\bar{a}(\bar{\theta}, \bar{r})}\right) \stackrel{\text { def }}{=}(\overline{\bar{\theta}}, \overline{\bar{r}})
$$

is a $C^{l}$-diffeomorphism from $A_{\bar{\delta}}$ onto its image, and $H_{2}\left(A_{\bar{\delta}}\right)$ contains an annulus $\mathrm{A}_{\delta}$, with $\overline{\bar{\delta}}>0$. The map $\overline{\bar{f}}^{\prime}=\mathrm{H}_{2} \circ \bar{f}^{\prime} \circ \boldsymbol{H}_{2}^{-1}:(\overline{\bar{\theta}}, \bar{r}) \mapsto\left(\overline{\bar{\Theta}}^{\prime}, \overline{\bar{R}}^{\prime}\right)$ still satisfies the monotone twist condition, for $\overline{\bar{\theta}}=\theta$, and the following formulae hold:

$$
\left\{\begin{array}{l}
\overline{\bar{\Theta}^{\prime}}=\overline{\bar{\theta}}+\omega+\overline{\bar{r}} \overline{\bar{a}}(\overline{\bar{\theta}}, \overline{\bar{r}}) \quad(\bmod 1) \\
\overline{\bar{R}}^{\prime}=\bar{\lambda} \bar{r}+w(\overline{\bar{\theta}}, \overline{\bar{r}})
\end{array}\right.
$$

with $\bar{a}=\bar{a} \circ H_{2}^{-1}$ and

\section{Denoting}

$$
w=\left[v+\left(\frac{u}{\bar{a}}\right) \circ \overline{f^{\prime}}-\bar{\lambda} \frac{u}{\bar{a}}+\bar{r}^{2} b\right] \circ H_{2}^{-1} .
$$

and

$$
\begin{aligned}
\bar{a}_{-} & =\min \bar{a}, \\
B & =\|b\|_{C^{0}}
\end{aligned}
$$

$$
V=\sup \left(\|u\|_{C^{0}},\|v\|_{C^{0}}\right)
$$

for each $\delta^{\prime} \leq \overline{\bar{\delta}}$, we dispose of the majoration:

$$
\|w\|_{C^{0}\left(\mathrm{~A}_{B^{\prime}}\right)} \leq V\left(1+\frac{1+\bar{\lambda}}{\bar{a}_{-}}\right)+\left(\delta^{\prime}+\frac{V}{\bar{a}_{-}}\right)^{2} B .
$$

Replacing $f$ and $f^{\prime}$ by their inverses and $\theta$ by $-\theta$ changes $\bar{\lambda}$ into its inverse and keeps the monotone twist condition, hence we can assume that $\bar{\lambda} \geq 1$. Using proposition 2 of $\S 5$, we finally obtain the following result:

THEOREM 1. Suppose that we are in the setting described above; let $\Gamma$ be the graph of the function $\psi$ introduced in 6.1, and assume that $K$ is an Aubry-Mather set of $f^{\prime}$ whose rotation number is $\omega$. Then one can find positive constants $\varepsilon, d$ and $C$ which do not depend on $f^{\prime}$, such that, if:

$$
\left\|f^{\prime}-f\right\|_{C^{1}} \leq \varepsilon
$$

and

$$
d(K, \Gamma)=\overline{\sup }_{x \in K} d(x, \Gamma) \leq d
$$


then

with

$$
d(K, \Gamma) \leq C\left\|f^{\prime}-f\right\|_{C^{0}}^{\tau}
$$

$$
\tau=\frac{2+\beta}{3+2 \beta} \text {. }
$$

Indeed, choose $d=\bar{\delta} / \max \mu$ and $\delta^{\prime}=d(K, \Gamma)$. Notice that the closeness of $f$ and $f^{\prime}$, together with the closeness of $K$ and $\Gamma$ imply that there are lifts $\tilde{f}$ and $\tilde{f}^{\prime}$ of $f$ and $f^{\prime}$ to $\mathbb{R} \times[-\delta, \delta]$ such that the rotation numbers of $\tilde{f}_{\mid \tilde{\Gamma}}$ and of $\tilde{f}_{\mid \tilde{K}}^{\prime}$ are equal in $\mathbb{R}$ (and not only modulo 1 ), where $\tilde{\Gamma}$ and $\tilde{K}$ are the preimages of $\Gamma$ and $K$ in $\mathbb{R} \times[-\delta, \delta]$.

Remark. The constant $C$ can be improved by using Proposition 1 instead of Proposition 2 if one knows that $K$ is an invariant curve such that $f_{\mid K}^{\prime}$ is $C^{\prime}$-conjugated to the rigid rotation $\theta \mapsto \theta+\omega$ by a map which is $C^{1}$-close to the projection $(\theta, r) \mapsto \theta$.

\section{Differentiable dependence}

7.1 .

We suppose, in this section, that we are given two monotone twist imbeddings $f$ and $f^{\prime}$ of some annulus $\mathbb{A}_{\delta}$ into $A$. We assume that $f$ is $C^{k}, k \in \mathbb{R}_{+}$possesses an invariant curve $\Gamma$ of class $C^{k}$, whose rotation number $\omega$ satisfies a diophantine condition with the exponent $2+\beta$ and constant $\gamma$ :

$$
\forall \frac{p}{q} \in \mathbb{Q} / \mathbb{Z}, \quad\left\|\omega-\frac{p}{q}\right\| \geq \frac{\gamma}{q^{2+\beta}}
$$

and that the restriction $f_{\mid \Gamma}$ is $C^{k}$-conjugated to the rigid rotation $\theta \mapsto \theta+\omega$.

In other words, there exist mappings $\psi \in C^{k}\left(\mathbb{T}^{1}\right)$ and $h \in \operatorname{Diff}_{+}^{k}\left(\mathbb{T}^{1}\right)$ such that $\|\psi\|_{C^{0}<\delta}$ and:

$$
f(h(\theta), \psi \circ h(\theta))=(h(\theta+\omega), \psi \circ h(\theta+\omega))
$$

holds for every $\theta \in \mathbb{T}^{1}$.

We also assume that $f^{\prime}$ is $C^{l}, 1 \leq l \leq k$, and has an Aubry-Mather set $K$ whose rotation number is $\omega$.

7.2 .

Theorem 2. Suppose that $k>5+3 \beta$ and that $l=k-2-\beta>3+2 \beta$. There exists $a$ linear continuous mapping $\Lambda:\left[C^{l}\left(\mathbb{A}_{\delta}\right)\right]^{2} \rightarrow C^{l-1-\beta}\left(\mathbb{T}^{1}\right)$ depending only on $f, \psi, h$ and $\omega$, and positive constants $\varepsilon<\frac{1}{2}, d, C$ and $\alpha$ such that, if:

and

$$
\left\|f^{\prime}-f\right\|_{c^{i}}<\varepsilon
$$

$$
d(K, \Gamma)=\sup _{x \in K} d(x, \Gamma)<d,
$$

then

$$
d\left(K, \Gamma^{\prime}\right)=\sup _{x \in K} d\left(x, \Gamma^{\prime}\right)<C\left\|f^{\prime}-f\right\|_{C^{\prime}}^{1+\alpha},
$$

where $\Gamma^{\prime}$ is the graph of $\psi+\Lambda \cdot\left(\tilde{f}^{\prime}-\tilde{f}\right)$, and where $\tilde{f}$ and $\tilde{f}^{\prime}$ are respective lifts of $f$ 
and $f^{\prime}$, chosen so that they satisfy the inequality:

$$
\left\|\tilde{f}^{\prime}-\tilde{f}\right\|_{C^{0}<\frac{1}{2}}
$$

7.3 .

Remarks. 1. This means that $K$ depends differentiably on $f^{\prime}$ at the point $f$.

2. The exponent $1+\alpha$ can be chosen equal to $2 \tau$, where $\tau=(2+\beta) /(3+2 \beta)>\frac{1}{2}$.

3. It is also possible to estimate the size of the "holes" of the Cantor set $K$ (whenever it is so), to get a majoration of the Hausdorff distance between $K$ and $\Gamma^{\prime}$ (see Herman [8]).

4. If one requires larger values of $k$ and $l$, then it is possible to get, with the same method, but using more normal forms, a Taylor expansion of arbitrary order for the location of $K$.

5. If $K$ is an invariant curve and if $f_{\mid K}^{\prime}$ is $C^{1}$-conjugated to the rigid rotation by a map which is $C^{1}$-close to the identity, then one can improve the constant $C$ by using Proposition 1 instead of Proposition 2 at the end of the proof.

6. The given values of $k$ and $l$ are not optimal (see next section).

7. Such results can be obtained for the curves whose existence is provided by Kolmogorov-Arnol'd-Moser theorem, when using the implicit function theorem in tame Frechet spaces (see Zehnder [15], Herman [6] and Bost [2]).

\section{4 .}

Proof. Like in $\S 6.1$, we may assume that $\psi=0$ and $h=\mathrm{Id}_{\mathrm{T}^{1}}$. Indeed, the graph transformation mapping induced by the diffeomorphism $H$ defined in 6.1 is twice differentiable in $C^{0}\left(T^{1}\right)$, since $H$ is $C^{k}$ and $k>2$ (this remark will be detailed at the end of the proof).

Therefore, we may write $f(\theta, r)=(\Theta, R)$, with:

$$
\left\{\begin{array}{l}
\Theta=\theta+\omega+r a(\theta, r) \quad(\bmod 1) \\
R=r \lambda(\theta, r)
\end{array}\right.
$$

the functions $a$ and $\lambda$ being positive and of class $C^{k-1}$.

Now, $f^{\prime}$ takes the following form:

and

$$
f^{\prime}(\theta, r)=\left(\Theta^{\prime}, R^{\prime}\right)
$$

$$
\left\{\begin{array}{l}
\Theta^{\prime}=\theta+\omega+r a(\theta, r)+u(\theta, r) \quad(\bmod 1) \\
R^{\prime}=r \lambda(\theta, r)+v(\theta, r),
\end{array}\right.
$$

where $u$ and $v$ belong to $C^{\prime}\left(A_{\delta}\right)$, and $u$ is determined uniquely by $\|u\|_{C^{0}<\frac{1}{2}}$.

Replacing $f$ and $f^{\prime}$ by their inverses and $\theta$ by $-\theta$, if needed, allows us to assume that:

$$
\log \bar{\lambda}=\int_{0}^{1} \log \lambda(\theta, 0) d \theta \geq 0,
$$

(see $\$ 6.3$ ).

7.5 .

The first change of variables we shall make is slightly different from the combination of the two changes of variables described in $\S 6$. Let $\mu \in C^{l}\left(\mathbb{T}^{1}\right)$ be the positive 
function defined by:

$$
\log \mu(\theta+\omega)-\log \mu(\theta)=\log \bar{\lambda}(\theta, 0)-\log \bar{\lambda}
$$

and

$$
\int_{0}^{1} \log \mu(\theta) d \theta=0
$$

(see Lemma 6.2).

We then set:

$$
\bar{a}(\theta)=a(\theta, 0) \mu(\theta)
$$

and define the $C^{\prime}$-map $H_{1}:(\theta, r) \mapsto(\bar{\theta}, \bar{r})$ by:

$$
\left\{\begin{array}{l}
\bar{\theta}=\theta \\
\bar{r}=\frac{1}{\bar{a}(\theta)}(r a(\theta, r)+u(\theta, r)) .
\end{array}\right.
$$

As soon as the $C^{1}$-norm of $u$ is sufficiently small (i.e. $\varepsilon$ is small enough), $H_{1}$ is a diffeomorphism and its image $H_{1}\left(\mathbb{A}_{\delta}\right)$ contains an annulus $A_{\tilde{\delta}}, \bar{\delta}>0$. The map:

$$
\begin{aligned}
\bar{f}^{\prime}=H_{1} \circ f^{\prime} \circ H_{1}^{-1}: & \mathbb{A}_{\bar{\delta}} \rightarrow \mathbb{A} \\
(\bar{\theta}, \bar{r}) & \mapsto\left(\bar{\Theta}^{\prime}, \bar{R}^{\prime}\right)
\end{aligned}
$$

is then given by the formulae:

$$
\left\{\begin{array}{l}
\bar{\Theta}^{\prime}=\bar{\theta}+\omega+\bar{r} \bar{a}(\theta) \quad(\bmod 1) \\
\bar{R}^{\prime}=\bar{\lambda} \bar{r}-\bar{v}(\bar{\theta}, \bar{r})
\end{array}\right.
$$

where $\bar{v}$ is $C^{l}$ and satisfies:

$$
\bar{v} \circ H_{1}=\left(\lambda \frac{a \circ f^{\prime}}{\bar{a} \circ f^{\prime}} \frac{\bar{a}}{a}-\bar{\lambda}\right) \bar{r}+\frac{u \circ f^{\prime}}{\bar{a} \circ f^{\prime}}-\frac{\lambda}{a} \frac{a \circ f^{\prime}}{\bar{a} \circ f^{\prime}} u+\frac{a \circ f^{\prime}}{\bar{a} \circ f^{\prime}} v
$$

(here, $\bar{r}$ has to be considered as a function of $\theta$ and $r$ ).

7.6.

The second change of variables being more complicated, we shall introduce simplified notations. When $s: \mathbb{T}^{1} \rightarrow \mathbb{R}$, we set:

$$
\begin{gathered}
{[s]=\int_{0}^{1} s(\theta) d \theta,} \\
s_{\omega}: \mathbb{T}^{1} \rightarrow \mathbb{R}, \theta \mapsto s(\theta+\omega), \\
s_{2 \omega}=\left(s_{\omega}\right)_{\omega}: \theta \mapsto s(\theta+2 \omega), \text { etc } \ldots
\end{gathered}
$$

and if $s: \mathbb{A}_{\bar{\delta}} \rightarrow \mathbb{R}$, then:

$$
\begin{gathered}
s_{0}: \mathbb{T}^{1} \rightarrow \mathbb{R}, \theta \mapsto s(\theta, 0) \\
s_{\omega}=\left(s_{0}\right)_{\omega}: \theta \mapsto s(\theta+\omega, 0), \text { etc } \ldots .
\end{gathered}
$$

A map $s: \mathbb{T}^{1} \rightarrow \mathbb{R}$ will also be regarded as a map from $A$ to $\mathbb{R}$, not depending on the second variable.

If a little letter, $s$ for instance, represents a function from $\mathbb{T}^{1}$ or $A_{\bar{\delta}}$ to $\mathbb{R}$, then the corresponding capital letter $S$ will represent the function $s \circ f^{\prime}$ (or $s \circ \Theta^{\prime}$ if $\left.s: \mathbb{T}^{1} \rightarrow \mathbb{R}\right)$, defined on $A_{\bar{\delta}}$ (even if $s$ is defined on $\mathbb{T}^{1}$ ). 
Let $\eta \in C^{l-1-\beta}\left(\mathbb{T}^{1}\right)$ and $\xi \in C^{l-2-2 \beta}\left(\mathbb{T}^{1}\right)$ be defined by:

and

$$
\eta_{\omega}-\bar{\lambda} \eta=-\frac{v_{0}}{\mu_{\omega}}+\left[\frac{v_{0}}{\mu_{\omega}}\right], \quad[\eta]=0
$$

where:

$$
\xi_{\omega}-\xi=-u_{0}+\bar{a}(\eta+c)
$$

$$
c=[\bar{a}]^{-1}\left[u_{0}-\bar{a} \eta\right] \text { is a constant. }
$$

We set $H_{2}(\bar{\theta}, \bar{r})=(\overline{\bar{\theta}}, \bar{r})$, with:

$$
\left\{\begin{array}{l}
\overline{\bar{\theta}}=\bar{\theta}+\xi(\bar{\theta}) \\
\bar{r}=\bar{r}+\frac{\xi(\bar{\Theta})-\xi(\bar{\theta})}{\bar{a}(\bar{\theta})} .
\end{array}\right.
$$

As $l>3+2 \beta$, this map is a diffeomorphism from $\mathbb{A}_{\bar{\delta}}$ onto its image $H_{2}\left(\mathbb{A}_{\bar{\delta}}\right)$ which contains an annulus $A_{\delta}$, provided that the constant $\varepsilon$ is small enough.

$$
\text { Let } \begin{aligned}
\bar{f}^{\prime}=H_{2} \circ \bar{f}^{\prime} \circ H_{2}^{-1}: \mathbb{A}_{\bar{\delta}} \rightarrow \mathbb{A}, \\
(\overline{\bar{\theta}}, \overline{\bar{r}}) \mapsto\left(\bar{\Theta}^{\prime}, \overline{\bar{R}}^{\prime}\right) .
\end{aligned}
$$

It is easy to compute that:

$$
\left\{\begin{array}{l}
\overline{\bar{\Theta}^{\prime}}=\overline{\bar{\theta}}+\omega+\overline{\bar{r}} \overline{\bar{a}}(\overline{\bar{\theta}}) \\
\overline{\bar{R}}^{\prime}=\bar{\lambda} \overline{\bar{r}}+\overline{\bar{v}}(\overline{\bar{\theta}}, \overline{\bar{r}}),
\end{array}\right.
$$

with $\overline{\bar{a}}(\bar{\theta}+\xi(\bar{\theta}))=\bar{a}(\bar{\theta})$ (hence $\overline{\bar{a}}$ is a positive function having the same minimum and maximum as $\bar{a})$ and

$$
\overline{\bar{v}}=\left(\lambda \frac{A}{\bar{A}} \frac{\bar{a}}{a}-\bar{\lambda}\right) \bar{r}+\frac{U}{\bar{A}}-\lambda \frac{A}{\bar{A}} \frac{u}{a}+\frac{A}{\bar{A}} v+\frac{\Xi-\Xi}{\bar{A}}-\bar{\lambda} \frac{\Xi-\xi}{\bar{a}}
$$

where $\Xi=\Xi \circ \bar{f}^{\prime}$.

In this heavy formula, we voluntarily omitted to specify the variables, as we are only interested in the $C^{0}$-norm of $\overline{\bar{v}}$. Actually, to be perfectly correct, when a function is defined in the $(\bar{\theta}, \bar{r})$ variables (for instance $\bar{a}, \bar{r}, \xi)$, one should compose it on the right by $H_{2}^{-1}$, and when it is defined in the $(\theta, r)$ variables (as $\lambda, a, u, v \ldots$ ), it should be composed by $H_{1}^{-1} \circ H_{2}^{-1}$.

To estimate the norm $\|\overline{\bar{v}}\|_{C^{0}}$, we shall decompose $\overline{\bar{v}}$ into different terms that make the functional equations satisfied by $\mu, \xi$ and $\eta$ appear.

$$
\begin{aligned}
\overline{\bar{v}}= & \left(\frac{\Xi}{\overline{\bar{A}}}-\frac{\xi_{2 \omega}}{\bar{a}_{\omega}}\right)-\left(\frac{\Xi}{\overline{\bar{A}}}-\frac{\xi_{\omega}}{\bar{a}_{\omega}}\right)-\bar{\lambda}\left(\frac{\Xi-\xi_{\omega}}{\bar{a}}\right) \\
& +(1-\bar{\lambda}) c+\left[\frac{v_{0}}{\mu_{\omega}}\right] \\
& +\left(\frac{A}{\bar{A}} v-\frac{a_{\omega}}{\bar{a}_{\omega}} v_{0}\right) \\
& +\left(\frac{U}{\bar{A}}-\frac{u_{\omega}}{\bar{a}_{\omega}}\right)+\frac{\bar{\lambda}}{\bar{a}}\left(u_{0}-u\right)-\left(\lambda \frac{A}{\bar{A}} \frac{\bar{a}}{a}-\lambda_{0} \frac{a_{\omega}}{\bar{a}_{\omega}} \frac{\bar{a}}{a_{0}}\right) \frac{u}{\bar{a}} \\
& +\left(\lambda \frac{A}{\bar{A}} \frac{\bar{a}}{a}-\lambda_{0} \frac{a_{\omega}}{\bar{a}_{\omega}} \frac{\bar{a}}{\bar{a}_{0}}\right) \bar{r} .
\end{aligned}
$$


7.7.

In the following inequalities, $C$ will denote a generic constant, depending only on $\omega, a$ and $\lambda$ (namely, on $\beta, \gamma, \min a, \min \lambda,\|a\|_{C^{\prime}}$ and $\|\lambda\|_{C^{\prime}}$ ). If we set

$$
M=\left\|f^{\prime}-f\right\|_{C^{\prime}}=\sup \left(\|u\|_{C^{\prime}},\|v\|_{C^{\prime}}\right),
$$

then we have, using the inequality of Lemma 6.2 , and the fact that $l=k-2-\beta>$ $3+2 \beta$, the majorations:

$$
\left\{\begin{array}{l}
\|\mu\|_{C^{\prime}} \leq C \\
\min \mu \geq C^{-1} \\
1 \leq \bar{\lambda} \leq C \\
\|\eta\|_{C^{l-1-\beta}} \leq C M \\
\|\xi\|_{C^{\prime}} \leq C M,
\end{array}\right.
$$

(for the last two inequalities, we use the fact that, if $s \in C^{j}\left(\mathbb{T}^{1}\right)$, then $\|s-[s]\|_{C^{j}} \leq$ $\left.\|s\|_{C^{j}}\right)$. Moreover,

$$
\begin{aligned}
|\bar{r}| \leq \bar{\delta} & \leq C(\delta+M), \\
\overline{\bar{\delta}} & \leq C(\delta+M) .
\end{aligned}
$$

Therefore,

$$
\|\overline{\bar{v}}-w\|_{c^{0} \leq C(\delta+M)^{2}}
$$

where

$$
w=(1-\bar{\lambda}) c+\left[\frac{v_{0}}{\mu_{\omega}}\right]
$$

is a constant.

Nevertheless, we need to majorate $\|\bar{v}\|_{C^{0}}$. For this purpose, we shall prove that $\overline{\bar{v}}$ vanishes, and thus, that:

$$
\|\overline{\bar{v}}\|_{c^{0}} \leq 2\|\overline{\bar{v}}-w\|_{c^{0}}
$$

Suppose that $\overline{\bar{v}}$ does not vanish and, for instance, that it is positive. Let $\overline{\bar{v}}_{-}=\min \overline{\bar{v}}$, and let $\left(\theta_{k}, r_{k}\right)_{k \in Z}$ be an orbit of $f^{\prime}$ belonging to $K$, and

$$
\left(\overline{\bar{\theta}}_{k}, \overline{\bar{r}}_{k}\right)=H_{2} \circ H_{1}\left(\theta_{k}, r_{k}\right) \text {. }
$$

If $\bar{\lambda}=1$, then:

$$
\overline{\bar{r}}_{k} \geq \overline{\bar{r}}_{k-1}+\overline{\bar{v}}_{-} \geq \overline{\bar{r}}_{0}+k \overline{\bar{v}}_{-}
$$

forbidding the orbit to stay in $A_{\delta}$ for all time; however, it should be so if $c$ and $d$ are small enough.

If $\bar{\lambda}>1$, then, by the same argument, as soon as some $\overline{\bar{r}}_{k_{0}}$ satisfies:

$$
\overline{\bar{r}}_{k_{0}}>-\frac{\overline{\bar{v}}_{-}}{\lambda-1},
$$

the sequence $\left(\overline{\bar{F}}_{k}\right)_{k \geq k_{0}}$ cannot stay bounded by $\overline{\bar{\delta}}$. Therefore,

$$
\overline{\bar{r}}_{k} \leq-\frac{\overline{\bar{v}}_{-}}{\lambda-1}
$$

holds for every integer $k$. But this is in contradiction with the fact that the rotation 
number of $K$ is $\omega$. Indeed, let $\tilde{f}$ and $\tilde{f}^{\prime}$ be respective lifts of $f$ and $f^{\prime}$ to $\tilde{A}_{\delta}=\mathbb{R} \times[-\delta, \delta]$ such that:

holds for every $\tilde{\theta} \in \mathbb{R}$ and:

$$
\tilde{f}(\tilde{\theta}, 0)=(\tilde{\theta}+w, 0)
$$

$$
\left\|\tilde{f}-\tilde{f}^{\prime}\right\|_{c^{\prime}} \leq c<\frac{1}{2}
$$

If $c$ and $d$ are small enough, then the rotation number of $\tilde{f}_{\mid K}^{\prime}$ (where $\tilde{K}$ is the preimage of $K$ in $\left.\tilde{A}_{\delta}\right)$ is strictly between $\omega-1$ and $\omega+1$, thus is equal to $\omega$.

Let now $\left(\tilde{\bar{\theta}}_{k}, \bar{F}_{k}\right)_{k \in Z} \in \tilde{A}_{\delta}^{Z}$ be an orbit of $\tilde{f}^{\prime}$ whose projection of $A_{\delta}$ is the prescribed orbit $\left(\overline{\bar{\theta}}_{k}, \overline{\bar{r}}_{k}\right)_{k \in \mathbf{Z}}$. The inequality (7.7.3) implies that:

$$
\tilde{\overline{\bar{\theta}}}_{k+1}-\tilde{\overline{\bar{\theta}}}_{k} \leq \omega-\frac{\overline{\bar{v}}_{-}}{\overline{\bar{\lambda}}-1} \min \bar{a},
$$

hence the rotation number of that orbit cannot exceed that quantity smaller than $\omega$.

We finally conclude that:

$$
\|\overline{\bar{v}}\|_{c^{0}} \leq C(\delta+M)^{2} \text {. }
$$

Denoting $\overline{\bar{K}}=H_{2} \circ H_{1}(K)$, we know from Proposition 2, that the distance $d\left(\bar{K}, A_{0}\right)$ between $\overline{\bar{K}}$ and $A_{0}$ satisfies:

$$
d\left(\overline{\bar{K}}, \mathrm{~A}_{0}\right) \leq C\|\overline{\bar{v}}\|_{C^{0}} \leq C(\delta+M)^{2 \tau},
$$

where $\tau=(2+\beta) /(3+2 \beta)$.

We can now shrink the annuli $A_{\delta}$ and $A_{\delta}$, so that

and

$$
\bar{\delta}=d\left(\bar{K}, \mathbb{A}_{0}\right)
$$

$$
\delta=C(\overline{\bar{\delta}}+M) \text {. }
$$

Hence we get:

$$
\overline{\bar{\delta}} \leq C(\overline{\bar{\delta}}+M)^{2 \tau},
$$

which implies, since $2 \tau>1$ :

$$
\overline{\bar{\delta}} \leq C M^{2+}
$$

as soon as $\overline{\bar{\delta}}$ is small enough, condition that can be insured by the sufficient smallness of $d$ and $c$.

7.8 .

Let now $\Gamma^{\prime \prime}=H_{1}^{-1} \circ H_{2}^{-1}\left(\mathbb{A}_{0}\right)$. If $c$ is small enough, then $\Gamma^{\prime \prime}$ is the graph of a function $\psi^{\prime \prime} \in C^{l}\left(\mathbb{T}^{1}\right)$ close to the zero function, which satisfies, for every $\theta \in \mathbb{J}^{1}$ :

$$
\psi^{\prime \prime} a\left(\theta, \psi^{\prime \prime}\right)=\xi(\theta)-\xi\left(\theta+\omega+\psi^{\prime \prime} a\left(\theta, \psi^{\prime \prime}\right)\right)-u\left(\theta, \psi^{\prime \prime}\right),
$$

(where $\psi^{\prime \prime}$ holds for $\psi^{\prime \prime}(\theta)$ ).

We define the linear operator $\Lambda$ by:

$$
\Lambda \cdot(u, v)=\mu(\eta+c)=\psi^{\prime}
$$

where we recall that:

$$
\begin{gathered}
\bar{\lambda} \frac{\mu_{\omega}}{\mu}=\lambda_{0}, \\
\eta_{\omega}-\bar{\lambda} \eta=-\frac{v_{0}}{\mu_{\omega}}+\left[\frac{v_{0}}{\mu_{\omega}}\right], \quad[\eta]=0,
\end{gathered}
$$


and

$$
c=\left[a_{0} \mu\right]^{-1}\left[u_{0}-a_{0} \mu \eta\right]
$$

(these simplified notations are defined in 7.6). The map $\psi^{\prime}$ then satisfies:

$$
\psi^{\prime} a_{0}=\xi-\xi_{\omega}-u_{0}
$$

As it is easily seen, we have:

$$
\|\xi\|_{C^{\prime}} \leq C M
$$

and

$$
\left\|\psi^{\prime \prime}\right\|_{C^{0}} \leq C M
$$

hence

$$
\left\|\psi^{\prime \prime}-\psi^{\prime}\right\|_{C^{0}} \leq C M^{2}
$$

If $\Gamma^{\prime}$ is the graph of $\psi^{\prime}$, then

$$
\begin{aligned}
d\left(K, \Gamma^{\prime}\right) & \leq d\left(K, \Gamma^{\prime \prime}\right)+d\left(\Gamma^{\prime \prime}, \Gamma^{\prime}\right) \\
& \leq C M^{2 \tau}+C M^{2} \\
& \leq C M^{2 \tau}
\end{aligned}
$$

since $\tau<1$.

In the general case where we do not assume that $\psi \equiv 0$ and $h=\operatorname{Id}_{\mathbf{T}^{1}}$, we proceed as follows. Let

$$
\begin{aligned}
f(\theta, r) & =(\Theta, R) \\
f^{\prime}(\theta, r) & =\left(\Theta^{\prime}, R^{\prime}\right)=(\Theta+u, R+v) .
\end{aligned}
$$

Let also $H:(\theta, r) \mapsto(\bar{\theta}, r)$ be defined by:

and

$$
\left\{\begin{array}{l}
\bar{\theta}=h^{-1}(\theta) \\
\bar{r}=r-\psi(\theta)
\end{array}\right.
$$

$$
\begin{aligned}
& \bar{f}=H \circ f \circ H^{-1}:(\bar{\theta}, \bar{r}) \mapsto(\bar{\Theta}, \bar{R}) \\
& \bar{f}^{\prime}=H \circ f^{\prime} \circ H^{-1}:(\bar{\theta}, \bar{r}) \mapsto\left(\bar{\Theta}^{\prime}, \bar{R}^{\prime}\right) .
\end{aligned}
$$

We have:

$$
\begin{aligned}
& \bar{u}=\bar{\Theta}^{\prime}-\bar{\Theta}=h^{-1}(\Theta+u)-h^{-1}(\Theta)=D h^{-1}(\Theta) u+O\left(u^{2}\right) \\
& \bar{v}=\bar{R}^{\prime}-\bar{R}=v+\psi(\Theta)-\psi(\Theta+u)=v-D \psi(\Theta) u+O\left(u^{2}\right),
\end{aligned}
$$

since $h$ and $\psi$ are $C^{2}$.

Let us replace, in the previous notations, $\Gamma^{\prime}$ by $\bar{\Gamma}^{\prime}$ and $\psi^{\prime}$ by $\bar{\psi}^{\prime}$. The set $\Gamma_{1}^{\prime}=H^{-1}\left(\bar{\Gamma}^{\prime}\right)$ then is the graph of $\psi_{1}^{\prime}=\psi+\bar{\psi}^{\prime} \circ h^{-1}$, which depends in an affine way on $\bar{u}$ and $\bar{v}$. Let us also denote

$$
\bar{\Lambda}:(\bar{u}, \bar{v}) \mapsto \bar{\psi}^{\prime}
$$

and

$$
\Lambda:(u, v) \mapsto \bar{\Lambda}\left(D h^{-1}(\Theta) u, v-D \psi(\Theta) u\right) \circ h^{-1} .
$$

The function $\psi^{\prime}=\Lambda \cdot(u, v)$ then satisfies

$$
\left\|\psi^{\prime}+\psi-\psi_{1}^{\prime}\right\|_{C^{0}} \leq C\left\|f-f^{\prime}\right\|_{C^{0}}^{2} .
$$


Therefore, the distance between the graph $\Gamma^{\prime}$ of $\psi+\psi^{\prime}$ and $\Gamma_{1}^{\prime}$ is bounded by $C\left\|f-f^{\prime}\right\|_{C^{0}}^{2}$, and thus, the distance from $K$ to $\Gamma^{\prime}$ is itself bounded by $C\left\|f-f^{\prime}\right\|_{C^{\prime}}^{2 \tau^{\prime}}$.

\section{Smoothing operators}

8.1.

We shall show, in this section, how the use of "smoothing operators" in our changes of variables can improve sensibly the minimal values of $k$ and $l$ allowed in Theorem 2. More precisely, when we have to solve a "difference equation" such as in Lemma 6.2 , instead of using an exact solution, we shall choose an approximative one, and estimate the error then made.

These smoothing operators are a one-parameter-family of operators:

$$
S_{t}: C^{0}\left(\mathbb{T}^{1}\right) \rightarrow C^{\infty}\left(\mathbb{T}^{1}\right)
$$

defined, for $t \geq 1$, as follows. Let $\hat{\chi}: \mathbb{R} \rightarrow \mathbb{R}$ be an even smooth function with compact support and identically equal to 1 in some neighborhood of 0 , and let:

$$
\chi(x)=\int_{-\infty}^{\infty} \hat{\chi}(y) e^{2 i \pi x y} d y
$$

The function $\chi$ is real-analytic, belongs to the space $\mathscr{S}$ of Schwartz (i.e. is fast decreasing at $\infty$, together with its derivatives) and satisfies:

$$
\begin{aligned}
& \int_{-\infty}^{\infty} \chi(x) d x=\hat{\chi}(0)=1 \\
& \int_{-\infty}^{\infty} x^{k} \chi(x) d x=(-2 i \pi)^{-k} \hat{\chi}^{(k)}(0)=0 .
\end{aligned}
$$

We then set, for $\varphi \in C^{0}\left(\mathbb{T}^{1}\right)$,

$$
S_{i} \varphi(\theta)=\int_{-\infty}^{\infty} t_{\chi}(t x) \varphi(\theta-x) d x
$$

It is not hard to see that $S_{1} \varphi$ is 1 -periodic, real-analytic and satisfies the following inequalities:

$$
\left\|S_{1} \varphi\right\|_{C^{k_{1}}} \leqq C_{k_{1}, k_{2}} t^{k_{1}-k_{2}}\|\varphi\|_{C^{k_{2}}}
$$

if $k_{1} \geq k_{2}$ and $\varphi \in C^{k_{2}}\left(\mathbb{T}^{1}\right)^{\dagger}$,

$$
\left\|S_{t} \varphi-\varphi\right\|_{C^{k_{1}}} \leq C_{k_{1}, k_{2}} t^{k_{1}-k_{2}}\|\varphi\|_{C^{k_{2}}}
$$

if $k_{1} \leq k_{2}$ and $\varphi \in C^{k_{2}}\left(T^{1}\right)$, where $k_{1}$ and $k_{2}$ are any positive real numbers, and $C_{k_{1}, k_{2}}$ are constants depending only on $k_{1}$ and $k_{2}$. One can also check the following identities (resulting from the commutativity of the convolution product):

$$
\begin{aligned}
\left(S_{t} \varphi\right)_{\omega} & =S_{t}\left(\varphi_{\omega}\right), \\
D\left(S_{t} \varphi\right) & =S_{t}(D \varphi), \\
{\left[S_{t} \varphi\right] } & =[\varphi] .
\end{aligned}
$$

${ }^{+}$Notice that (8.1.1) can be modified in an inequality which is valuable for all $k_{1}$ and $k_{2}$ :

$$
\left\|S_{1} \varphi\right\|_{k_{1}} \leq C_{k_{1}, k_{2}}\left(1+t^{k_{1}-k_{2}}\right)\|\varphi\|_{k_{2}} \text {. }
$$


8.2.

We shall now make use of these smoothing operators in the definition of the function $\mu, \eta$ and $\xi$ introduced in $\S 7$. Let $x, y$ and $z$ be real numbers greater than 1 , whose values will be precised later on; we set, instead of (7.5.1), (7.6.1) and (7.6.2):

$$
\begin{gathered}
(\log \mu)_{\omega}-\log \mu=S_{x}\left(\log \lambda_{0}\right)-\log \bar{\lambda} \\
\eta_{\omega}-\bar{\lambda} \eta=-S_{y}\left(\frac{v_{0}}{\mu_{\omega}}\right)+\left[\frac{v_{0}}{\mu_{\omega}}\right] \\
\xi_{\omega}-\xi=S_{z}\left(-u_{0}+\bar{a}(\eta+c)\right)
\end{gathered}
$$

so that no assumption needs to be made on $k$ on $l$.

We shall still assume that:

$$
[\log \mu]=[\eta]=0
$$

and that the constant $c$ is defined by (7.6.3) (with the new definition of $\bar{a}=a_{0} \mu$ and $\eta$ ).

The new expression of $\overline{\bar{v}}$ is then obtained from the old one (7.6.3) by adding a certain number of error terms:

$$
\begin{aligned}
\overline{\bar{v}}= & \left(\frac{\Xi}{\overline{\bar{A}}}-\frac{\xi_{2 \omega}}{\bar{a}_{\omega}}\right)-\left(\frac{\Xi}{\bar{A}}-\frac{\xi_{\omega}}{\bar{a}_{\omega}}\right)-\bar{\lambda}\left(\frac{\Xi-\xi_{\omega}}{\bar{a}}\right) \\
& +\frac{1}{\bar{a}_{\omega}}\left[S_{z}(\bar{a}(\eta+c))_{\omega}-\bar{a}_{\omega}\left(\eta_{\omega}+c\right)\right)-\frac{\bar{\lambda}}{\bar{a}}\left(S_{z}(\bar{a}(\eta+c))-\bar{a}(\eta+c)\right) \\
& -\frac{1}{\bar{a}_{\omega}}\left(S_{z} u_{\omega}-u_{\omega}\right)+\frac{\bar{\lambda}}{\bar{a}}\left(S_{z} u_{0}-u_{0}\right)+(1-\bar{\lambda}) c+\left[\frac{v_{0}}{\mu_{\omega}}\right] \\
& -\left(S_{y}\left(\frac{v_{0}}{\mu_{\omega}}\right)-\frac{v_{0}}{\mu_{\omega}}\right)+\left(\frac{A}{\bar{A}} v-\frac{a_{\omega}}{\bar{a}_{\omega}} v_{0}\right) \\
& +\left(\frac{U}{\bar{A}}-\frac{u_{\omega}}{\bar{a}_{\omega}}\right)-\bar{\lambda}\left(\frac{u-u_{0}}{\bar{a}}\right)+\left(\lambda \frac{A}{\bar{A}} \frac{\bar{a}}{a}-\lambda_{0} \frac{a_{\omega}}{\bar{a}_{\omega}} \frac{\bar{a}}{a_{0}}\right)\left(\bar{r}-\frac{u}{\bar{a}}\right) \\
& +\bar{\lambda}\left(\exp \left(\log \lambda_{0}-S_{x} \log \lambda_{0}\right)-1\right)\left(\bar{r}-\frac{u}{\bar{a}}\right) .
\end{aligned}
$$

As in $\S 7.7$, the point is to get a majoration of $\|\bar{v}\|_{C^{0}}$ looking like (7.7.4):

$$
\|\overline{\tilde{v}}\|_{C^{0}} \leq C(\delta+M)^{\sigma}
$$

with an exponent $\sigma$ which we need to satisfy:

$$
\sigma>\frac{1}{\tau}, \quad \tau=(2+\beta) /(3+2 \beta)
$$

(we recall that $M=\left\|f^{\prime}-f\right\|_{C^{\prime}}$ ). The inequalities (6.2.1), (8.1.1) and (8.1.2) will naturally be of importance.

8.3.

Let us set:

$$
\bar{M}=\delta+M \text {. }
$$

We have to assume that $k>3+\beta$ and $l>1$, so that the following quantities are bounded by a constant $C$ depending only on $f, \omega, k$ and $l$ : 


$$
\|a\|_{C^{k-1}},\left\|\frac{1}{a}\right\|_{C^{0}},\|\lambda\|_{C^{k-1}},\|\log \lambda\|_{C^{k-1}}, \bar{\lambda},\|\mu\|_{C^{1}},\left\|\frac{1}{\mu}\right\|_{C^{0^{0}}},\|a\|_{C^{1}},\left\|\frac{1}{\bar{a}}\right\|_{C^{0}} ;
$$

while the following ones are bounded by $C \bar{M}$ :

$$
\|u\|_{C^{\prime}},\|v\|_{C^{\prime}},|\bar{r}|,\left\|\Theta^{\prime}-\theta-\omega\right\|_{C^{o}},\left\|\Theta^{\prime}-\theta-2 \omega\right\|_{C^{0}}
$$

where $\Theta^{\prime}=\theta \circ f^{\prime}$ and $\Theta^{\prime}=\theta \circ f^{\prime 2}$.

Consequently, if $w$ is the constant defined in (7.7.2), there exists a constant $C$ not depending on $f^{\prime}$ such that:

$$
\begin{aligned}
C^{-1}\|\overline{\bar{v}}-w\|_{C^{0}} \leq & \|\xi\|_{C^{1}} \bar{M}+\left\|S_{z}(\bar{a}(\eta+c))-\bar{a}(\eta+c)\right\|_{C^{0}} \\
& +\left\|S_{z} u_{0}-u_{0}\right\| c_{C^{0}}+\left\|S_{y}\left(\frac{v_{0}}{\mu_{\omega}}\right)-\frac{v_{0}}{\mu_{\omega}}\right\|_{C^{0}} \\
& +\bar{M}^{2}+\left\|S_{x}\left(\log \lambda_{0}\right)-\log \lambda_{0}\right\|_{C^{0}} \bar{M} .
\end{aligned}
$$

Again, as $\overline{\bar{v}}$ must vanish, we know that:

$$
\left\|\left.v\right|_{c^{0}} \leq 2\right\| \overline{\bar{v}}-w \|_{c^{0}} .
$$

Our aim is now to estimate each term of the second member of (8.3.1).

In the following estimations, the letter $C$ will denote a generic constant depending only $f, \omega, k, l$ and $j$, while $j$ will be any non-negative real number (bounded in fact by $\max (k, l)$ so that $C$ does not depend on $j)$.

Majoration of $\|\xi\|_{C^{1}}$ will come from the definition of $\xi$ (see (8.2.3)) and what we know about difference equations (see Lemma 6.2) and smoothing operators (see (8.1.1) and footnote).

For this purpose, we first need to estimate the norms of $\mu$ and $\eta$, using (8.2.1) and (8.2.2), and of $S_{z} u_{0}$. One has:

$$
\|\mu\|_{C^{j}} \leq C\left(1+x^{2+\beta+j-k}\right) .
$$

Therefore:

$$
\left\|\frac{v_{0}}{\mu_{\omega}}\right\|_{C^{\prime}} \leq C \bar{M}\left(1+x^{2+\beta+l-k}\right)
$$

and

$$
\begin{aligned}
\|\eta\|_{C^{\prime}} & \leq C\left\|\frac{v_{0}}{\mu_{\omega}}\right\|_{C^{\prime}}\left(1+y^{1+\beta+j-l}\right) \\
& \leq C\left(1+x^{2+\beta+l-k}\right)\left(1+y^{1+\beta+j-l}\right) \bar{M} .
\end{aligned}
$$

Moreover:

$$
\left\|S_{z} u_{0}\right\| C^{j} \leq C\left(1+z^{j-l}\right) \bar{M} .
$$

These three inequalities lead to the following majoration of $\|\xi\|_{C^{1}}$.

$$
\begin{aligned}
& \|\xi\|_{C^{1}} \leq C\left(\|\mu \eta\|_{C^{2+\beta}}+\left\|S_{z} u_{0}\right\|_{C^{2+\beta}}\right) \\
& \leq C\left(\|\mu\|_{C^{2+\beta}}\|\eta\|_{C^{0}}+\|\mu\|_{C^{0}}\|\eta\|_{C^{2+\beta}}+\left\|S_{z} u_{0}\right\|_{C^{2+\beta}}\right) \\
& \leq C \bar{M}\left(1+x^{4+2 \beta-k}+x^{7+4 \beta-2 k}+y^{3+2 \beta-1}+x^{2+\beta+1-k} y^{3+2 \beta-1}+z^{2+\beta-1}\right) \text {. }
\end{aligned}
$$

The second term of (8.3.1) is treated by using the second inequality of smoothing operators (8.1.2) and what we already know about $\mu$ and $\eta$, that is (8.3.3) and (8.3.4). From the definition of the constant $c$ (see (7.6.3)), the function $-u_{0}+\bar{a}(\eta+c)$ 
has zero mean value on the circle. Therefore, it must vanish, and $\eta+c$ has to take values bounded by $C \bar{M}$. Hence, one has:

$$
\|\eta+c\|_{C^{j}} \leq 2\|\eta\|_{C^{j}}+C \bar{M}
$$

Consequently,

$$
\begin{aligned}
\left\|S_{z}(\bar{a}(\eta+c))-\bar{a}(\eta+c)\right\|_{C^{0}} & \leq C\|\mu(\eta+c)\|_{C^{k-1} z^{1-k}} \\
& \leq C\left(\|\mu\|_{C^{k-1}}\|\eta+c\|_{C^{0}}+\|\mu\|_{C^{0}}\|\eta+c\|_{\left.C^{k-1}\right) z^{1-k}}\right. \\
& \leq C \bar{M} z^{1-k}\left(x^{1+\beta}+x^{4+3 \beta-k}+y^{\beta+k-l}+x^{2+\beta+l-k} y^{\beta+k-l}\right) .
\end{aligned}
$$

The last terms of (8.3.1) are simply estimated through (8.1.2):

$$
\begin{aligned}
\| S_{z} u_{0}-u_{0} & \|_{C^{0}} \leq C \bar{M} z^{-l} \\
\left\|S_{y}\left(\frac{v_{0}}{\mu_{\omega}}\right)-\frac{v_{0}}{\mu_{\omega}}\right\|_{C^{0}} & \leq C\left\|\frac{v_{0}}{\mu_{\omega}}\right\|_{C^{1}} y^{-l} \\
& \leq C \bar{M} y^{-l}\left(1+x^{2+\beta+l-k}\right) ;
\end{aligned}
$$

and

$$
\left\|S_{x} \log \lambda_{0}-\log \lambda_{0}\right\|_{C^{0}} \leq C x^{1-k}
$$

8.4.

Let us now assume that $\bar{M}<1$, and set

$$
x=\bar{M}^{-X}, \quad y=\bar{M}^{-Y}, \quad z=\bar{M}^{-Z},
$$

where $X, Y$ and $Z$ are positive numbers to be fixed later on.

In order to obtain a majoration of the type (8.2.5), we need that the quantity $1 / \tau=(3+2 \beta) /(2+\beta)$ be strictly bounded from above by the following quantities (following (8.3.1) to $(8.3 .10)$ :

$$
\begin{aligned}
& Q_{1}=2 \\
& Q_{2}=2-X(4+2 \beta-k) \\
& Q_{3}=2-X(7+4 \beta-2 k) \\
& Q_{4}=2-Y(3+2 \beta-l) \\
& Q_{5}=2-X(2+\beta+l-k)-Y(3+2 \beta-l) \\
& Q_{6}=2-Z(2+\beta-l) \\
& Q_{7}=1-X(1+\beta)+Z(k-1) \\
& Q_{8}=1-X(4+3 \beta-k)+Z(k-1) \\
& Q_{9}=1-Y(\beta+k-l)+Z(k-1) \\
& Q_{10}=1-X(2+\beta+l-k)-Y(\beta+k-l)+Z(k-1) \\
& Q_{11}=1+l Z \\
& Q_{12}=1+l Y \\
& Q_{13}=1-X(2+\beta+l-k)+l Y \\
& Q_{14}=1+X(k-1) \text {. } \\
& \left.\begin{array}{l}
\left\{\begin{array}{l}
\text { also from the fifth } \\
\text { term of }(8.3 .2) \\
\text { from }(8.3 .6)
\end{array}\right. \\
\text { from (8.3.7) } \\
\text { from (8.3.8) } \\
\text { from (8.3.9) } \\
\text { from (8.3.10) }
\end{array}\right\}
\end{aligned}
$$


We recall that:

$$
\frac{1}{\tau}=1+\frac{1+\beta}{2+\beta}=2-\frac{1}{2+\beta} \in(1,2) .
$$

From $Q_{14}>1 / \tau$, we get:

$$
X>\frac{1+\beta}{(2+\beta)(k-1)} .
$$

From $Q_{12}, Q_{13}>1 / \tau$, we get:

$$
\begin{cases}Y>\frac{1+\beta}{(2+\beta) l} & \text { if } k \geq 2+\beta+l \\ Y>\frac{(1+\beta)(1+\beta+l)}{(2+\beta) l(k-1)} & \text { if } k \leq 2+\beta+l .\end{cases}
$$

From $Q_{7}, \ldots, Q_{11}>1 / \tau$, we get:

$$
\begin{cases}Z>\frac{(1+\beta)(k+\beta)}{(2+\beta) l(k-1)} & \text { if } k \geq 2+\beta+l \\ Z>\frac{(1+\beta)(1+\beta+l)(k+\beta)}{(2+\beta) l(k-1)^{2}} & \text { if } k \leq 2+\beta+l\end{cases}
$$

The inequality:

$$
Q_{1}>1 / \tau
$$

is always satisfied, while $Q_{2}, Q_{3}>1 / \tau$ can be satisfied by a good choice of $X$ if, and only if:

$$
k>\frac{8+11 \beta+4 \beta^{2}}{3+2 \beta}=2+2 \beta+\frac{2+\beta}{3+2 \beta}=k_{\beta}>2 .
$$

The minoration of $Q_{4}$ by $1 / \tau$ can be obtained from a good choice of $Y$, when $k \geq l+2+\beta$, as soon as:

$$
l>\frac{3+5 \beta+\beta^{2}}{2+\beta}=2+\beta+\frac{\beta-1}{2+\beta}=l_{\beta},
$$

and then, it implies $Q_{5}>1 / \tau$.

On the other hand, if $k \leq l+2+\beta$, then $Q_{4} \geq Q_{5}$, and $Q_{5}>1 / \tau$ can be satisfied if:

$$
k>2+2 \beta+\frac{1}{2+\beta}+\frac{(1+\beta)^{2}(3+2 \beta)}{(2+\beta) l} .
$$

Then, it is not hard to see that if $k$ and $l$ satisfy the above inequalities, the condition $Q_{6}>1 / \tau$ can always be fulfilled by a choice of $Z$ compatible with (8.4.4). One can also check that (8.4.5) and (8.4.6) imply (8.4.7) when $\beta \geq \beta_{0}$, where $\beta_{0}$ is the only positive root of the polynomial:

$$
4 \beta^{5}+27 \beta^{4}+66 \beta^{3}+64 \beta^{2}+16 \beta-3
$$

(one has: $\beta_{0} \approx 0,1245 \cdots<\frac{1}{8}$ ).

We are finally lead to the following result: 
THEOREM 2'. If $\beta \geq \beta_{0}$, theorem 2 (see 7.2) is available when $k>k_{\beta}$ and $l>l_{\beta}$, where $k_{\beta}$ and $l_{\beta}$ are defined in (8.4.5) and (8.4.6).

When $\beta<\beta_{0}$, then one has to add the extra condition (8.4.7).

The following picture indicates the set of allowed values of $(k, l)$ for $\beta=0$. Of course, the Remark 7.3.2 is now false, and if one picks a value of $(k, l)$ close to the boundary of this set, then the number $\alpha$ will be extremely small.

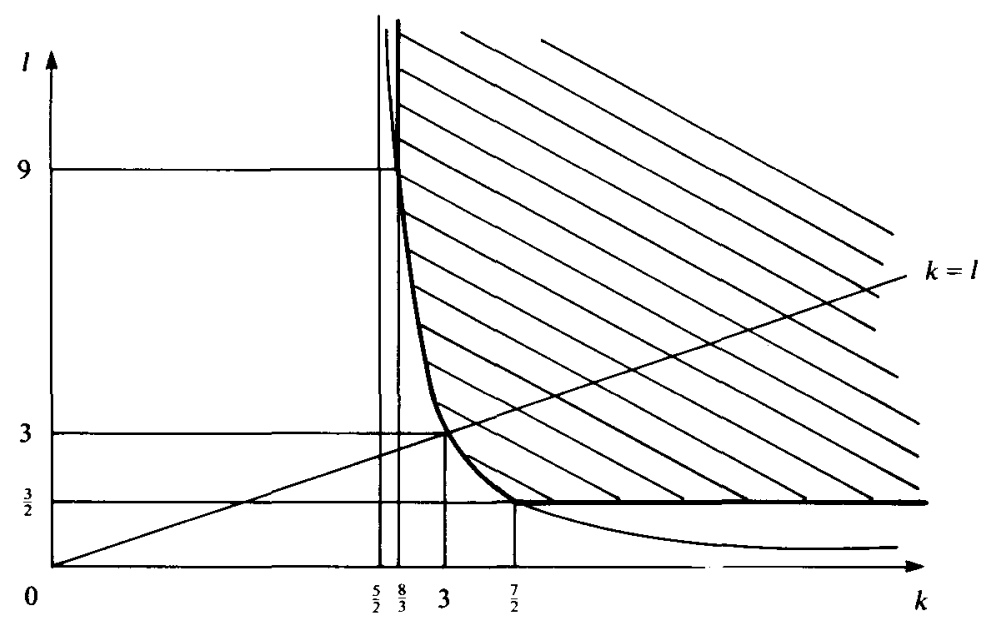

\section{Separation of invariant curves}

9.1.

We consider an annulus $A_{\delta}, \delta>0$ and an orientation preserving $C^{k}$-imbedding:

$$
\begin{aligned}
f: \mathbb{A}_{\delta} & \rightarrow \mathbb{A} \\
(\theta, r) & \mapsto(\Theta, R) .
\end{aligned}
$$

We assume that $f$ possesses an invariant curve $\Gamma$ which is the graph of a $C^{k}$. function $\psi$, and such that $f_{\mid \Gamma}$ is conjugated to a rigid rotation $\theta \mapsto \theta+\omega$ by a map $h \in \operatorname{Diff}_{+}^{k}\left(\mathbb{T}^{1}\right)$, where the rotation number $\omega \in \mathbb{T}^{1}$ satisfies a diophantine condition with exponent $2+\beta$ and constant $\gamma>0$;

$$
\forall \frac{p}{q} \in \mathbb{Q} / \mathbb{Z}, \quad\left\|\omega-\frac{p}{q}\right\| \geq \frac{\gamma}{q^{2+\beta}} .
$$

We saw in $\$ 6$ that, after an elementary change of coordinates, we can assume that $\Gamma=\mathbb{A}_{0}$ and that $f_{\mid \Gamma}$ acts on the first coordinate by the rigid rotation. Hence, there exist functions $a, \lambda \in C^{k-1}\left(\pi^{1}\right)$, the second one being positive everywhere (since $f$ preserves orientation), such that,

$$
\left\{\begin{array}{l}
\Theta=\theta+\omega+r a(\theta, r) \quad(\bmod 1) \\
R=r \lambda(\theta, r) .
\end{array}\right.
$$

Moreover, it is explained in 6.2 how, if $k>3+\beta$, then one can find new coordinates 
in which $f$ takes the form:

$$
\left\{\begin{array}{l}
\Theta=\theta+\omega+r a(\theta, r) \quad(\bmod 1) \\
R=\bar{\lambda} r+r^{2} b(\theta, r)
\end{array}\right.
$$

where, presently, $a$ is only of class $C^{k-2-\beta}$, while $b$ is $C^{k-3-\beta}$ (in particular, continuous). Actually, $a(\theta, r)$ may change of sign, since we did not make any monotone twist assumption on $f$. The constant $\bar{\lambda}$ is however positive.

Replacing if needed $f$ by its inverse $f^{-1}$, we shall suppose that $\bar{\lambda} \leq 1$.

9.2 .

THEOREM. Let $\omega^{\prime} \in \mathbb{T}^{1}$ be different from $\omega$, and $K$ be an Aubry-Mather set of $f$ with rotation number $\omega^{\prime}$. Then there exists a constant $c$ depending only on $\|a\|_{C^{\circ}}$ and $\|b\|_{C^{\circ}}$, such that:

$$
K \cap \mathbb{A}_{\delta^{\prime}}=\varnothing
$$

where

$$
\delta^{\prime}=c\left\|\omega^{\prime}-\omega\right\| .
$$

We recall that $\left\|\omega-\omega^{\prime}\right\|=\inf \left|\tilde{\omega}-\tilde{\omega}^{\prime}\right|$, the infimum being taken over the different lifts of $\omega$ and $\omega^{\prime}$ in $\mathbb{R}$.

Going back to the original coordinates, this means that the Aubry-Mather set $\mathrm{K}$ of $f$ cannot approach the invariant curve $\Gamma$ at a distance less than $c^{\prime}\left\|\omega^{\prime}-\omega\right\|$, the constant $c^{\prime}$ depending on $\|\psi\|_{C^{1}},\|h\|_{C^{\prime}}$ and $c$.

Remarks. 1. If $\bar{\lambda}<1$ then, actually, $\Gamma$ is an attractor and no Aubry-Mather set of $f$ can meet the bassin of $\Gamma$, whose width is of the order of $1-\bar{\lambda}$.

2. The constant $c$ only depends on the $C^{k}$-norm of $f, \psi$ and $h$, and on the exponent $2+\beta$ and the constant $\gamma$.

3. As $K$ is a minimal invariant compact set, it has to lie entirely above or entirely below $\mathbb{A}_{\delta^{\prime}}$, for $f$ preserves the upper and the lower semi-cylinder delimited by $A_{0}$. 4. We made no assumption on $\omega^{\prime}$ (except $\omega^{\prime} \neq \omega$ ). In particular, $\omega^{\prime}$ could be a rational and $K$ a periodic orbit.

5. Consider the set:

$$
\Delta_{\beta \gamma}=\left\{\omega \in \mathbb{T}^{1} / \forall q \in \mathbb{N}-\{0\}, \quad\|q \omega\| \geq \frac{\gamma}{q^{1+\beta}}\right\} .
$$

Consider also an annulus $A_{\delta}, \delta>0$ and a number $\delta_{1} \in(0, \delta)$. Let $f$ be a monotone twist map from $A_{\delta}$ to $A$ which has the intersection property (i.e. any curve $\mathscr{C}$ homotopic to $A_{0}$ meets its image $f(\mathscr{C})$ ). Assume that $f$ is smooth and close in some $C^{k}$-topology, $k>3+2 \beta$, to an integrable system:

$$
(\theta, t) \mapsto(\theta+t(r), r)
$$

where $t$ is a smooth function satisfying $d t / d r>0$.

By Kolmogorov-Arnol'd-Moser theorem (see [15]), $f$ possesses for each $\omega \in$ $\Delta_{\beta \gamma} \cap J, J=t\left(\left[-\delta_{1}, \delta_{1}\right]\right)$, an invariant curve $\Gamma_{\omega}$ which is the graph of a smooth function $\psi_{\omega}$ such that $f_{\mid \Gamma_{\omega}}$ is smoothly conjugated to the rotation $\theta \mapsto \theta+\omega$. 
The previous theorem asserts that the map

$$
\begin{gathered}
\psi: \mathbb{J}^{1} \times\left(\Delta_{\beta \gamma} \cap J\right) \rightarrow \mathbb{A}_{\delta} \\
(\theta, \omega) \mapsto\left(\theta, \psi_{\omega}(\theta)\right)
\end{gathered}
$$

Has a Lipschitz inverse (defined on the image of $\psi$ ) and, thus, that the union of the curves $\Gamma_{\omega}$ has positive measure as soon as $\Delta_{\beta \gamma} \cap J$ has itself positive measure (this is the case, for $\beta>0$ fixed, if $\gamma$ is small enough).

9.3.

Proof. Since $\mathrm{A}_{0}$ and $K$ are different minimal invariant compact sets for $f$, they cannot meet. Let $\left(\theta_{0}, r_{0}\right)$ be a point of $K$ minimizing $\left|r_{0}\right|$, and let:

be its orbit.

$$
\left(\theta_{k}, r_{k}\right)=f^{k}\left(\theta_{0}, r_{0}\right), \quad k \in \mathbb{Z}
$$

Set $A=\|a\|_{C^{0}}$ and $B=\|b\|_{C^{0}}$.

If $\left|r_{0}\right| \geq 1 / B$ then one has:

$$
\left|r_{0}\right| \geq \frac{2}{B}\left\|\omega-\omega^{\prime}\right\|
$$

and the conclusion of the theorem holds.

If $\left|r_{0}\right|<1 / B$ we shall assume, for instance, that $r_{0}>0$. By Remark 3, we know that $r_{k}>0$ for every $k$.

On the one hand,

$$
r_{k} \leq \bar{\lambda} r_{k-1}+r_{k-1}^{2} B
$$

hence, since $\bar{\lambda} \leq 1$, as long as $r_{k-1} \leq 1 / B$, one has:

$$
r_{k} \leq \frac{r_{k-1}}{1-r_{k-1} B}
$$

Thus, if $k \in \mathbb{N}$, and $k r_{0} B<1$, then:

$$
r_{k} \leq \frac{r_{0}}{1-k r_{0} B}
$$

On the other hand, let $\tilde{f}$ be a lift of $f$ to $\tilde{A}_{\delta}=\mathbb{R} \times[-\delta, \delta]$ so that, for $\tilde{\theta} \in \mathbb{R}$, we get:

$$
\tilde{f}(\tilde{\theta}, 0)=(\tilde{\theta}+\tilde{\omega}, 0)
$$

where $\tilde{\omega}$ is a lift of $\omega$. We shall also define $\tilde{K}$ as the preimage of $K$ in $\tilde{A}_{\delta}$ and $\tilde{\omega}^{\prime}$ as the rotation number of $\tilde{f}_{\mid \tilde{K}}$. Let $\tilde{\theta}_{0}$ be any lift of $\theta_{0}$ and:

$$
\left(\tilde{\theta}_{k}, r_{k}\right)=\tilde{f}^{k}\left(\tilde{\theta}_{0}, r_{0}\right)
$$

We may assume that $\tilde{\omega}^{\prime}>\tilde{\omega}$. Indeed, this latter assumption is independent of that made on $r_{0}$, since, to get $r_{0}>0$, we might need to replace $r$ and $-r$ and, to get $\tilde{\omega}^{\prime}>\tilde{\omega}$, we could have to change $\theta$ into $-\theta$, and each of these two modifications does not affect the fact that $f$ preserves orientation.

One has:

$$
\begin{aligned}
\tilde{\theta}_{k} & \leq \tilde{\theta}_{0}+k \tilde{\omega}+A\left(r_{0}+\cdots+r_{k-1}\right) \\
& \leq \tilde{\theta}_{0}+k \tilde{\omega}+\frac{A}{B} \log \left(1-k B r_{0}\right)^{-1}
\end{aligned}
$$

as long as $k B r_{0}<1$. 
If, furthermore, $k B r_{0} \leq \frac{1}{2}$, then:

$$
\tilde{\theta}_{k} \leq \tilde{\theta}_{0}+k \tilde{\omega}+k C r_{0}
$$

with $C=2 A \log 2$.

Let

$$
p / q \in \mathbb{Q} \cap\left[\frac{\omega+\omega^{\prime}}{2}, \omega^{\prime}\right]
$$

be such that:

$$
0<q \leq \frac{2}{\tilde{\omega}^{\prime}-\tilde{\omega}}+1 \leq \frac{3}{\left\|\omega^{\prime}-\omega\right\|}
$$

One has:

$$
q \tilde{\omega}^{\prime}-p>0
$$

hence

$$
\tilde{\theta}_{q}-\tilde{\theta}_{0}-p>0
$$

Thus, either:

$$
q B r_{0} \geq \frac{1}{2}
$$

or

$$
q C r_{0} \geq p-q \tilde{\omega} .
$$

In the first case, we get:

$$
r_{0} \geq \frac{\left\|\omega^{\prime}-\omega\right\|}{6 B}
$$

while, in the second one, the following holds:

$$
r_{0} \geq \frac{1}{C}\left(\frac{p}{q}-\tilde{\omega}\right) \geq \frac{\left\|\omega^{\prime}-\omega\right\|}{2 C} .
$$

9.4.

Consider now a smooth monotone twist $\operatorname{map} f: \mathbb{A}_{\delta} \rightarrow \mathbb{A}$ possessing a smooth invariant curve $\Gamma$ such that $f_{\mid \Gamma}$ is smoothly conjugated to the rigid rotation $\theta \rightarrow \theta+\omega$. We shall assume that $\Gamma$ is non normally hyperbolic (this occurs for instance when $f$ satisfies the intersection property), and that $\omega$ is diophantine with exponent $2+\beta$ and constant $\gamma$.

In suitable coordinates, one has:

$$
f(\theta, r)=(\Theta, R)
$$

with:

$$
\left\{\begin{array}{l}
\Theta=\theta+\omega+r a(\theta, r) \quad(\bmod 1) \\
R=r \lambda(\theta, r)
\end{array}\right.
$$

and, by assumption,

$$
\int_{0}^{1} \log \lambda(\theta, 0) d \theta=0
$$


Following Lemma 6.2, let the functions $\mu$ and $\nu$ (from $\mathbb{T}^{1}$ to $\mathbb{R}$ ) satisfy:

$$
\log \mu(\theta+\omega)-\log \mu(\theta)=\log \lambda(\theta, r)
$$

and

$$
\nu(\theta+\omega)-\nu(\theta)=-a(\theta, 0) \mu(\theta)+\int_{0}^{1} a(\theta, 0) \mu(\theta) d \theta .
$$

To be entirely determined, normalizations should be added, such as:

$$
\int_{0}^{1} \log \mu(\theta) d \theta=0
$$

and

$$
\int_{0}^{1} \nu(\theta) d \theta=0
$$

Let us now define $H:(\theta, r) \rightarrow(\bar{\theta}, \bar{r})$ by:

$$
\left\{\begin{array}{l}
\bar{\theta}=\theta+r \frac{\nu(\theta)}{\mu(\theta)} \quad(\bmod 1) \\
\bar{r}=r\left(a(\theta, r)+\lambda(\theta, r) \frac{\nu(\Theta)}{\mu(\Theta)}-\frac{\nu(\theta)}{\mu(\theta)}\right) .
\end{array}\right.
$$

An easy computation shows that the map:

$$
\bar{f}=H \circ f \circ H^{-1}:(\bar{\theta}, \bar{r}) \mapsto(\bar{\Theta}, \bar{R})
$$

takes the following form:

$$
\left\{\begin{array}{l}
\bar{\Theta}=\bar{\theta}+\omega+\bar{r} \quad(\bmod 1) \\
\bar{R}=\bar{r}+\bar{r}^{2} b(\bar{\theta}, \bar{r}) .
\end{array}\right.
$$

THEOREM 4. Let $K$ be an Aubry-Mather set of $f$ whose rotation number $\omega^{\prime}$ satisfies a diophantine condition with exponent $2+\beta^{\prime}$ and constant $\gamma^{\prime}$. There exist constants $d>0$ and $C$, depending only on $\beta^{\prime}, \gamma^{\prime}$ and $\|b\|_{C^{0}}$ such that, if:

$$
\left\|\omega^{\prime}-\omega\right\|<d
$$

and

$$
\sup _{x \in K} d(x, \Gamma)<d
$$

then

$$
\bar{K}=H(K) \subset \mathbb{V}^{1} \times\left[\Delta \omega-\delta^{\prime}, \Delta \omega+\delta^{\prime}\right],
$$

where

$$
\begin{aligned}
\omega^{\prime} & =\omega+\Delta \omega \quad(\bmod 1), \\
|\Delta \omega| & =\left\|\omega^{\prime}-\omega\right\|
\end{aligned}
$$

and

$$
\begin{aligned}
\delta^{\prime} & =C\left\|\omega^{\prime}-\omega\right\|^{2 \tau^{\prime}}, \\
\tau^{\prime} & =\frac{2+\beta^{\prime}}{3+2 \beta^{\prime}}>\frac{1}{2} .
\end{aligned}
$$


Remarks. 1. Finite differentiability is enough for that purpose. Namely, we need that $f$, together with $\Gamma$ and the conjugation of $f_{\mid \Gamma}$ to the rigid rotation, be $C^{k}$ with $k>4+2 \beta$.

2. This minimal smoothness can be reduced by the same method as in $\S 7$, but we then have to replace the exponent $2 \tau^{\prime}$ in the definition of $\delta^{\prime}$, by a number $1+\alpha^{\prime}>1$ that might be close to 1 .

3. Analogous results were obtained by Lazutkin [9] for the caustics of a billiard in a convex domain, and by Pöschel [12] for the invariant tori of a nearly integrable Hamiltonian system.

Proof. Let us set:

$$
\begin{aligned}
\bar{r}^{\prime} & =\bar{r}-\Delta \omega \\
\bar{R}^{\prime} & =\bar{R}-\Delta \omega .
\end{aligned}
$$

One has:

$$
\left\{\begin{array}{l}
\bar{\Theta}=\bar{\theta}+\omega^{\prime}+\bar{r}^{\prime} \\
\bar{R}^{\prime}=\bar{r}^{\prime}+\left(\bar{r}^{\prime}+\Delta \omega\right)^{2} b\left(\bar{\theta}+\bar{r}^{\prime}+\Delta \omega\right) .
\end{array}\right.
$$

Notice that if $\tilde{f}$ and $\tilde{\omega}$ are lifts of $f$ and $\omega$ such that:

$$
\tilde{f}(\tilde{\theta}, 0)=(\tilde{\theta}+\tilde{\omega}, 0)
$$

and if $\tilde{K}$ denotes the preimage of $K$ in $\mathbb{R} \times[-\delta, \delta]$, then, for $d$ small enough, the rotation number of $\tilde{f}_{\mid \tilde{K}}$ must be $\tilde{\omega}^{\prime}=\tilde{\omega}+\Delta \omega$.

Therefore, Proposition 2 (see $\S 5.2$ ) implies that, if one sets:

$$
\rho=\sup _{(\bar{\theta}, \bar{r}) \in \bar{K}}|\bar{r}-\Delta \omega|
$$

then there are constants $C$ and $C^{\prime}$ such that:

$$
\begin{aligned}
\rho & \leq C(\rho+|\Delta \omega|)^{2 \tau^{\prime}} \\
& \leq C^{\prime}\left(\rho^{2 \tau^{\prime}}+|\Delta \omega|^{2 \tau^{\prime}}\right) .
\end{aligned}
$$

Hence, if:

then:

$$
d<\left(2 C^{\prime}\right)^{1 /\left(2 \tau^{\prime}-1\right)}
$$

$$
\rho \leq 2 C^{\prime}|\Delta \omega|^{2 \tau^{\prime}} \text {. }
$$

\section{REFERENCES}

[1] S. Aubry \& P. Y. Le Daeron. The discrete Frenkel-Kontorova model and its extensions I. Physica $8 D$ (1983), 381-422.

[2] J-B. Bost. Tores invariants des systèmes dynamiques hamiltoniens. Séminaire Bourbaki 639 (Feb. 1985); Astérisque 133-134 (1986).

[3] A. Chenciner. La dynamique au voisinage d'un point fixe elliptique de Poincaré et Birkhoff à Aubry et Mather. Séminaire Bourbaki 622 (Feb. 1984), published in Astérisque 121-122 (1985) 147-170.

[4] G. Hedlund. Geodesics on a 2-dimensional riemannian manifold with periodic coefficients. Ann. Math. serie II 33 (1932).

[5] M. R. Herman. Sur la conjugation différentiable des difféọmorphismes du c̄ercle à des rotations. I.H.E.S. Pub. Math. 49 (1975), 5-234.

[6] M. R. Herman. Démonstration du théorème de la courbe translatée. Manuscript, 1980.

[7] M. R. Herman. Sur les courbes invariantes par les difféomorphismes de l'anneau. 1 Astérisque 103-104 (1983) Astérisque 144 (1986). 
[8] M. R. Herman. Remarque sur les courbes de Mather et Aubry. Conference at the séminaire de l'Ecole Polytechnique, 14/2/1983.

[9] V. F. Lazutkin. The existence of caustics for a billiard problem in a convexe domain. Math. USSR-Iszuestija 71 (1973), 185-214.

[10] J. Mather. Existence of quasi-periodic orbits for twist homeomorphisms. Topology 21, 4 (1982), 457-467.

[11] J. Moser. On invariant curves of area preserving mappings of an annulus. Nachr. Akad. Wiss. Göttingen (1962), 1-20.

[12] J. Pöschel. Integrability of Hamiltonian systems on Cantor sets. Comm. Pure Appl. Math. 35 (1982), 653-696.

[13] H. Rüssmann. On the existence of invariant curves of twist mappings of an annulus. Lect. Notes in Math. 1007 (1983), 677-718.

[14] J-C. Yoccoz. Conjugaison différentiable des difféomorphismes du cercle dont le nombre de rotation vérifie une condition diophantienne. Ann. Sci. E.N.S., 4e s. 17 (1984), 333-359.

[15] E. Zehnder. Generalized implicit function theorem with application to some small divisors problems II. Comm. in Pure Appl. Math. 29 (1976), 49-111. 